\title{
Rotational excitations of polar molecules on an optical lattice: from novel exciton physics to quantum simulation of new lattice models
}

\author{
Marina Litinskaya and Roman V. Krems \\ Department of Chemistry, University of British Columbia, Vancouver, V6T 1Z1, Canada
}

\begin{abstract}
Ultracold polar molecules trapped on an optical lattice is a many-body system that, under appropriate conditions, may support collective excitations reminiscent of excitons in solid state crystals. Here, we discuss the rotational excitations of molecules on an optical lattice leading to rotational Frenkel excitons. Apart from solid hydrogen, there is no other natural system that exhibits rotational excitons. The rotational excitons have unique properties that can be exploited for tuning non-linear exciton interactions and exciton-impurity scattering by applying an external electric field. We show that this can be used to explore the competing role of the dynamical and kinematic exciton-exciton interactions in excitonic energy transfer and to study quantum localization in a dynamically tunable disordered potential. The rotational excitons can also be used as a basis for quantum simulation of condensed matter models that cannot be realized with ultracold atoms. As an example, we discuss the possibility of engineering the Holstein model with polar molecules on an optical lattice.
\end{abstract}

\section{Introduction}

Ultracold atoms offer the possibility to study few- and many-body quantum systems with exquisite control over microscopic interactions. This has led to spectacular experiments [1], bridging different areas of physics. While the research directions stimulated by experiments with ultracold atoms are very diverse, one can identify several overarching trends that gained momentum in recent years. The most prominent of these trends is the effort aimed at understanding quantum phase transitions [2], leading to the experimental studies of BoseEinstein condensation (BEC) [3], bosonic superfluidity [4, quantum magnetism [5], manybody spin dynamics [6], Efimov states [7], Bardeen-Cooper-Schrieffer (BCS) superfluidity [8] and the BEC-BCS crossover 99. With the development of techniques for trapping atoms in periodic potentials of optical lattices [10] and single atom detection [11, ultracold atoms became an ideal platform for quantum simulation of lattice models used in solid state physics [12]. As demonstrated by the realization of the Mott insulator - superfluid transition with atoms trapped in a three-dimensional optical lattice [13], the experiments with ultracold atoms hold the promise of insight into the details of the Hubbard model, which may help unravel the mechanism of high- $T_{\mathrm{c}}$ superconductivity [14]. 
The development of experimental methods for the production of ultracold polar molecules [15] has widened the possibilities for quantum simulation of condensed matter models to a great extent. A combination of the rotational, spin and hyperfine degrees of freedom with the long-range dipolar interactions enabled by the dipole moment of molecules allows for engineering a great variety of lattice models that cannot be realized with atoms [16, 17]. Of particular interest is the possibility of creating quantum phases with topological order [18], which are resilient to perturbations preserving the topology and are, therefore, ideal for quantum computation. To this end, a major effort of current experiments is focused on preparing a dense ensemble of ultracold polar molecules trapped in optical lattices [19].

Another focus of research with quantum degenerate gases is on emergent phenomena, such as solitons [20], rotons [21], vortices [22], spin waves [23] and polarons [24]. These experiments aim to elucidate emergence in natural systems, and may potentially lead to the development of novel ultra-sensitive sensors of gravity and electromagnetic fields. While these studies cover a wide range of collective dynamics of ultracold atoms and molecules in a single quantum state, much less is known about the effect of internal degrees of freedom of ultracold particles. For example, the role of rotational transitions in the excitation spectrum of a molecular Bose-Einstein condensate had not been addressed until very recently [25]. Yet, the internal degrees of freedom can be used to explore new regimes of collective phenomena, especially in molecular systems that provide a dense spectrum of internal excitations [26, 27].

In this article, we consider rotational excitations of polar molecules trapped on an optical lattice. These excitations give rise to rotational excitons analogous to collective electronic excitations in molecular crystals. In contrast to excitons in natural solids, the properties of rotational excitons can be dynamically controlled by tuning the energy level structure of the trapped molecules, which can be used to study new regimes of Frenkel exciton physics not accessible in natural solid state crystals. For example, we show that non-linear interactions of excitons can be tuned to examine the competition between the dynamical and kinematic effects and demonstrate that rotational excitons can be used to study quantum localization in a dynamically tunable disordered potential. The rotational excitons can also be used as a basis for quantum simulation of condensed-matter models that cannot be realized with ultracold atoms. As an example, we discuss the possibility of engineering the Holstein model with polar molecules on an optical lattice. In order to present these results in the context of current research, we briefly describe the related work on quantum simulation of many-body Hamiltonians with ultracold atoms and molecules and the basics of Frenkel exciton physics.

\section{Quantum simulation of lattice models}

The difficulty of simulating a quantum many-body system on a classical computer increases exponentially with the number of quantum states. An alternative, currently at the focus of detailed research, is quantum simulation [28]. Quantum simulation involves the design of a controllable quantum system in order to simulate the properties of another, more complicated system (analog quantum simulator). This idea is generally attributed to Feynman [29], although quantum simulation is already mentioned in an earlier publication by Manin [30]. The key ingredient of quantum simulation is the mapping of the Hamiltonian of the simulated system onto the Hamiltonian of the simulator with controllable parameters. 
Tuning the parameters of the controlled system can then be used to map out the phase diagram of the simulated system. The recent literature offers many ideas about how to build quantum simulators based on ultracold quantum gases [31], ultracold trapped ions [32, single photon sources and detectors [33, ultracold Rydberg atoms 34, circuit QED [35], quantum dots and $\mathrm{N}-\mathrm{V}$ centers in diamond [36].

Ultracold atoms trapped in a periodic potential of overlapped laser beams (optical lattices) is considered as one of the most promising systems for quantum simulation of lattice models [10, 37]. The geometry and strength of the optical lattice potentials can be controlled by adjusting the laser intensity and the beam overlap angle, which enables control over the translational motion of atoms in the lattice [38]. The separation of the lattice sites in an optical lattice is equal to half the wavelength of the trapping laser field. Recent technological breakthroughs permit the detection of ultracold atoms with sub-wavelength resolution, allowing for single-site addressing [39].

\subsection{Lattice models}

The most prominent example of quantum simulation with ultracold atoms is the realization of the Hubbard model [40, 13]

$$
\hat{H}_{H}=-t \sum_{\sigma ;\langle n, m\rangle} \hat{c}_{n, \sigma}^{\dagger} \hat{c}_{m, \sigma}+\frac{U}{2} \sum_{n} \hat{n}_{n, \downarrow} \hat{n}_{n, \uparrow}+\sum_{\sigma ; n} E_{\sigma} \hat{n}_{n, \sigma}
$$

where $\sigma=\uparrow$ or $\downarrow, \hat{c}_{n, \sigma}^{\dagger}$ and $\hat{c}_{n, \sigma}$ are the creation and annihilation operators for fermions, satisfying the anti-commutation rule $\left\{\hat{c}_{n, \sigma}^{\dagger}, \hat{c}_{m, \sigma}\right\}=\delta_{n m}, \hat{n}_{n, \sigma}=\hat{c}_{n, \sigma}^{\dagger} \hat{c}_{n, \sigma}$ is the particle number operator, and $E_{\sigma}$ is the energy of particles in state $\sigma$. The angle brackets $\langle n, m\rangle$ indicate that the summation is over nearest neighbors only. In this simple form, the Hubbard Hamiltonian accounts for nearest neighbor tunnelling $(t)$ and on-site interactions for fermions in different spin states $(U)$. The fermions with the same spin experience hardcore repulsion. This model is widely used for the studies of high- $T_{\mathrm{c}}$ superconductivity [41] and quantum magnetism [42].

With bosonic atoms, it is possible to realize the Bose-Hubbard model:

$$
\hat{H}_{B H}=-t \sum_{\langle n, m\rangle} \hat{b}_{n}^{\dagger} \hat{b}_{m}+\frac{U}{2} \sum_{n} \hat{n}_{n}\left(\hat{n}_{n}-1\right)+E_{0} \sum_{n} \hat{n}_{n},
$$

where $\hat{b}_{n}^{\dagger}$ and $\hat{b}_{n}$ are the creation and annihilation operators for bosons, satisfying the commutation rule $\left[\hat{b}_{n}^{\dagger}, \hat{b}_{m}\right]=\delta_{n m}$, and $\hat{n}_{n}=\hat{b}_{n}^{\dagger} \hat{b}_{n}$ is the particle number operator. This model has been used in many studies of the properties of bosonic gases such as superfluidity [43]. If $U \ll t$, the particles are delocalized around the lattice and the ground state of the system is a superfluid. This Hamiltonian also allows for modelling the properties of fermionic systems when quantum statistics plays no role and the properties of the system are entirely determined by the relative efficiency of hopping (governed by $t$ ) and the interaction $(U)$. In particular, when $U \gg t$ (the on-site repulsion dominates over the inter-site hopping), this bosonic Hamiltonian reproduces transition to the Mott insulator phase [44, where the number of atoms per lattice site is fixed. This transition is typical for electrons in a metal. It is thus possible to realize the same physical phenomenon with particles satisfying different quantum statistics. We shall discuss this in detail in section 2.2 . 
Recent experiments demonstrated the possibility of creating the Mott insulator phase with ultracold atoms filling up to $99 \%$ of the lattice sites [45]. A major thrust of current research is to extend these experiments to ultracold molecules. The experiments with polar molecules in optical lattices offer the possibility of realizing lattice models with long-range interactions [16, 17, 46, 47. In the Bose-Hubbard model the inter-site interactions are accounted for by adding to the Hamiltonian (2) a term $\hat{H}_{V}=\sum_{\langle n, m\rangle} V_{n, m} \hat{n}_{n} \hat{n}_{m}$ describing density-density correlations. In the limit of the strong on-site repulsion, when $\left\langle\hat{n}_{n}\right\rangle=0$ or 1 , the system reduces to the $t-V$ model, described by the Hamiltonian

$$
\hat{H}_{t-V}=-t \sum_{\langle n, m\rangle} \hat{b}_{n}^{\dagger} \hat{b}_{m}+E_{0} \sum_{n} \hat{n}_{n}+V \sum_{\langle n, m\rangle} \hat{n}_{n} \hat{n}_{m}
$$

with the constraint

$$
\hat{b}_{n}^{\dagger} \hat{b}_{n}^{\dagger}|\Phi\rangle=\hat{b}_{n} \hat{b}_{n}|\Phi\rangle=0
$$

for any physical state of the crystal $|\Phi\rangle$, which accounts for the infinite on-site repulsion. With $V=0$ this Hamiltonian corresponds to the lattice analog of the Tonks-Girardeau gas [48, 49]. This model is equivalent to an anisotropic spin- $1 / 2 X X Z$ model [50].

In addition, molecules allow for the possibility of realizing a variety of lattice spin models [12], such as

$$
\hat{H}_{\sigma}=-\sum_{\langle n, m\rangle}\left(J_{x} \sigma_{n}^{x} \sigma_{m}^{x}+J_{y} \sigma_{n}^{y} \sigma_{m}^{y}+J_{z} \sigma_{n}^{z} \sigma_{m}^{z}\right)-g H \sum_{n} \sigma_{n}^{z}
$$

where $\sigma_{n}^{x, y, z}$ are the Pauli matrices for lattice site $n$. This is the so-called Heisenberg model. If the interactions are isotropic in the plane perpendicular to the $z$ axis, i.e. for $J_{x}=J_{y}$, this Hamiltonian reduces to the $X X Z$-model [51]. In the isotropic Heisenberg model, $J_{x}=J_{y}=J_{z}$. The $X Y$-model is for the case when $J_{z}=0$. The scalar version of the Heisenberg model $\left(J_{x}=J_{y}=0\right)$ is the Ising model. In the Ising model, spins are treated as scalars, which can take one of two values: $s_{n}= \pm 1$. The Hamiltonian becomes

$$
\hat{H}_{I}=-\sum_{n, m} J_{n m} s_{n} s_{m}-h \sum_{n} s_{n}
$$

The Ising model can be realized with ultracold molecules in the spin-less ${ }^{1} \Sigma$ electronic state trapped on an optical lattice in a Mott insulator phase with one molecule per lattice site. The rotational levels of ${ }^{1} \Sigma$ molecules in an external electric field form an isolated two-level system, illustrated in Figure 17. The ground $|g\rangle$ and excited $|e\rangle$ states can be used as the spin states $s_{n}$. The coupling constant $J_{n m}$ is determined by the dipole-dipole interaction between molecules in different lattice sites. The rotational excitation $|g\rangle \rightarrow|e\rangle$ thus leads to spin waves of the Ising model. In order to simulate more complicated spin models, such as Eq. (5), it is necessary to use molecules with more complex structure [16, 17].

\subsection{Bosons, Fermions and Paulions}

The Hamiltonians presented in section 2.1 describe very different physical systems, consisting of particles with all possible statistics (bosons, fermions, spin matrices and pseudospins). They are, however, mutually related. Some of these Hamiltonians can be mapped 
onto each other, even when the quantum statistics of mapping and mapped particles are different.

Consider an ensemble of arbitrary two-level systems on a lattice. The state of a twolevel system in lattice site $n$ can be characterized by the operators $\hat{p}_{n}^{\dagger}$ and $\hat{p}_{n}$ describing, respectively, the creation and destruction of the excited state. Operators for different lattice sites necessarily commute, as they act on different variables. On the other hand, the same site cannot accommodate more than one excitation, which is a feature of Fermi statistics. The commutation relations for the operators $\hat{p}_{n}$ are

$$
\begin{gathered}
\hat{p}_{n} \hat{p}_{m}^{\dagger}-\hat{p}_{m}^{\dagger} \hat{p}_{n}=0 \quad(n \neq m) \\
\hat{p}_{n} \hat{p}_{n}^{\dagger}+\hat{p}_{n}^{\dagger} \hat{p}_{n}=1 .
\end{gathered}
$$

The operators with such "mixed" statistics are called paulions (in condensed matter physics [52]) or hard-core bosons (in atomic physics [49]). Using the $\hat{p}$-operators, we can write the following general Hamiltonian

$$
\hat{H}_{p}=E_{0} \sum_{n} \hat{p}_{n}^{\dagger} \hat{p}_{n}+\sum_{n, m}^{\prime} t_{n, m} \hat{p}_{n}^{\dagger} \hat{p}_{m}+\sum_{n, m}^{\prime} V_{n, m} \hat{p}_{n}^{\dagger} \hat{p}_{m}^{\dagger} \hat{p}_{n} \hat{p}_{m}
$$

which is identical to the hard-core Hamiltonian (3) with the constraint (4) absorbed into the statistical properties of paulions (77). The prime over the sum symbols indicates that $n \neq m$. For $V=0$, i.e. when the density-density correlations are absent, the Hamiltonian (8) is identical to the Ising Hamiltonian (6) with $h=0$. This Hamiltonian is also used to model Frenkel excitons in solid state molecular crystals [53].

Any unitary transformation preserves the commutation properties of the bosonic and fermionic operators. For example, the Fourier transform of bosons must produce bosons. Therefore, many articles have been devoted to mapping paulions onto "effective" particles with bosonic or fermionic statistics. Girardeau showed [49] that in 1D the many-body wave function of hard-core bosons - paulions - corresponds (up to a sign) to a many-body wave function of a gas of fictitious non-interacting spin-less fermions. The exact mapping was proposed later by Chestnut and Suna for a 1D system in the nearest neighbor approximation [56]. This is a variant of the Jordan-Wigner transformation [54], and it works very well in 1D. However, in higher dimensions it may cause problems: the effective fermionic operators are non-local, i.e. the expression for the fermionic operators in site $n$ is dependent on the occupation numbers at other sites. That is why this transformation is not as effective in dimensions higher than one. Another possibility is to use the Agranovich-Toshich transformation [57], which expresses $\hat{p}$-operators through an infinite series of bosonic operators $\hat{b}_{n}$ and $\hat{b}_{n}^{\dagger}$, in a way ensuring that for any number of bosons per lattice site $\hat{N}_{n}^{(b)}=\hat{b}_{n}^{\dagger} \hat{b}_{n}=0,1,2,3 \ldots$ the eigenvalues of the number operator for paulions $\hat{N}_{n}=\hat{p}_{n}^{\dagger} \hat{p}_{n}$ are only 1 or 0 , so that the unphysical states with $\hat{N}_{n}>1$ do not occur. Then the paulionic Hamiltonian (8) reduces to a sum of terms consisting of the same number of creation and annihilation bosonic operators, which we schematically denote by $\hat{H}_{\nu}=\sum_{\{n, m\}} \alpha_{\nu} \hat{b}_{n_{1}}^{\dagger} \ldots \hat{b}_{n_{\nu}}^{\dagger} \hat{b}_{m_{1}} \ldots \hat{b}_{m_{\nu}} ; \nu=1 \ldots \infty$. Keeping the pairwise interactions only and

\footnotetext{
${ }^{1}$ We note that similar transformations exist also for spin matrices $\sigma_{n}^{+}=\sigma_{n}^{x}+i \sigma_{n}^{y}, \sigma_{n}^{-}=\sigma_{n}^{x}-i \sigma_{n}^{y}$ and $\sigma_{n}^{z}$. They can be mapped onto fermionic operators by the Jordan-Wigner transformation [54, and onto bosonic operators by the Holstein-Primakoff transformation [55].
} 
assuming that $|t| \ll E_{0}$, one obtains:

$$
\hat{H}=E_{0} \sum_{n} \hat{b}_{n}^{\dagger} \hat{b}_{n}+\sum_{n} t_{n, m} \hat{b}_{n}^{\dagger} \hat{b}_{m}+\sum_{n, m}^{\prime} V_{n, m} \hat{b}_{n}^{\dagger} \hat{b}_{m}^{\dagger} \hat{b}_{n} \hat{b}_{m}+\hat{H}_{\mathrm{kin}},
$$

where

$$
\hat{H}_{\text {kin }}=-2 E_{0} \sum_{n=1}^{\mathcal{N}} \hat{b}_{n}^{\dagger} \hat{b}_{n}^{\dagger} \hat{b}_{n} \hat{b}_{n} .
$$

Thus, the Agranovich-Toshich transformation reduces the pauilonic (hard-core boson) Hamiltonian to a Hamiltonian describing a gas of bosons interacting via delta-like pairwise interaction with the strength $2 E_{0}$. Higher-order corrections to the interaction energy can be obtained by including the omitted terms. The Hamiltonian $\hat{H}_{\text {kin }}$ describes the kinematic interaction, which we discuss in section 4.1. In contrast to the Wigner-Jordan transformation, this approach is very effective in $3 \mathrm{D}$, where $\hat{H}_{\text {kin }}$ can be treated as a perturbation. For $2 \mathrm{D}$ systems, the delta-like scattering with the magnitude $2 E_{0}$, which is the largest energy scale of the problem, is very strong and does not allow for a perturbative treatment [58]. In $1 \mathrm{D}$, the effect of scattering is even stronger and the transformation to bosons makes no sense at all. To summarize, in 1D paulions are well described by a gas of fermions, in 3D by bosons, in 2D they are something in between. Finally, we note that in principle it is not necessary to use any of these transformations; one can work directly with paulions taking into account their commutation relations (7).

\section{Rotational Frenkel excitons}

While the majority of research with atoms and molecules on optical lattices has so far focused on quantum transport of particles in a lattice potential or quantum simulation of lattice models, such as the ones described above, the ability to control the structure of molecules trapped on an optical lattice can also be exploited to study quantum energy transport and collective excitations reminiscent of excitons in solid state crystals. In this section, we discuss the formation of rotational excitons. These excitons have unique properties and can, in turn, be used as a basis for quantum simulation of new physical phenomena and new lattice models that cannot be realized with atoms on an optical lattice. This is discussed in sections $4-6$.

Rotational excitation of polar molecules trapped on an optical lattice in the Mott insulator phase with one molecule per site gives rise to the formation of rotational Frenkel excitons [59]. The Frenkel exciton is a charge-less quasiparticle, which describes the excitation transfer in molecular crystals [60. Here, we consider the transition between the absolute ground state $|g\rangle$ of trapped polar molecules and their first rotational excited state. We assume that the molecules reside in the ground vibrational state of the ground electronic state ${ }^{1} \Sigma$. In the presence of a dc electric field $\mathcal{E}_{f}$, the three-fold degeneracy of the rotationally excited state $\left|N=1, M_{N}\right\rangle$ is lifted and the state with the projection $M_{N}=0$ of the total angular momentum is detuned from the states with $M_{N}= \pm 1$ (Figure 1a). We assume that the detuning is large enough so that the latter can be disregarded and consider the isolated two-level system of the state $|g\rangle$ and the rotational excited state with $M_{N}=0$, denoted by $|e\rangle$. 
The molecular states are the eigenstates of the Hamiltonian

$$
\hat{H}_{n}^{(\mathrm{mol})}=B_{e} \hat{N}_{n}^{2}-\hat{\mathbf{d}}_{n} \cdot \mathcal{E}_{f},
$$

where $B_{e}$ is the rotational constant, $\hat{N}_{n}$ is the operator of the angular momentum, and $\hat{\mathbf{d}}_{n}$ is the dipole moment of the molecule in site $n$. The field-dressed states are linear combinations of the field-free rotational states

$$
\begin{aligned}
& |g\rangle=\sum_{N} \alpha_{N}\left(\mathcal{E}_{f}\right)\left|N, M_{N}=0\right\rangle, \\
& |e\rangle=\sum_{N} \beta_{N}\left(\mathcal{E}_{f}\right)\left|N, M_{N}=0\right\rangle .
\end{aligned}
$$

The coefficients $\alpha_{N}$ and $\beta_{N}$ are determined by the electric field strength $\mathcal{E}_{f}$. In the limit $\mathcal{E}_{f} \rightarrow 0$, the states $|g\rangle$ and $|e\rangle$ become, respectively, $\left|N=0, M_{N}=0\right\rangle$ and $\left|N=1, M_{N}=0\right\rangle$.

It is convenient to introduce the transition operators $\hat{P}_{n}$ defined by the equations $\hat{P}_{n}^{\dagger}\left|g_{m}\right\rangle=\delta_{n m}\left|e_{n}\right\rangle$ and $\hat{P}_{n}\left|e_{m}\right\rangle=\delta_{n m}\left|g_{n}\right\rangle$. As discussed in section 2.2, these operators describe hard-core bosons, or paulions, and the excitonic Hamiltonian describes a hardcore boson gas with long-range interactions, which is equivalent to the Ising model. The rotational excitons can thus be mapped onto other systems described by the $t-V$ model, or the Ising model.

Using the $\hat{P}_{n}$-operators, the total Hamiltonian for $\mathcal{N}$ molecules on an optical lattice can be written as [53]

$$
\hat{H}_{\text {tot }}=\hat{H}_{\text {exc }}+\hat{H}_{\text {dyn }}+\hat{H}_{\mathrm{n}-\mathrm{c}},
$$

where the first term accounts for the excitation transfer between lattice sites

$$
\hat{H}_{\text {exc }}=\sum_{n} E_{0} \hat{P}_{n}^{\dagger} \hat{P}_{n}+\sum_{n, m}^{\prime} J(n-m) \hat{P}_{n}^{\dagger} \hat{P}_{m}
$$

with the constant $J(n-m)$ determined by the matrix elements of the inter-molecular excitation transfer due to dipole-dipole interaction

$$
J(n-m)=\left\langle e_{n} g_{m}\left|\hat{V}_{d d}(n-m)\right| g_{n} e_{m}\right\rangle .
$$

The second term describes non-linear interactions between excitons:

$$
\hat{H}_{\mathrm{dyn}}=\frac{1}{2} \sum_{n, m}^{\prime} D(n-m) \hat{P}_{n}^{\dagger} \hat{P}_{m}^{\dagger} \hat{P}_{n} \hat{P}_{m},
$$

with the interaction constant $D(n-m)$ determined by

$$
\begin{aligned}
& D(n-m)=\left\langle e_{n} e_{m}\left|\hat{V}_{d d}(n-m)\right| e_{n} e_{m}\right\rangle+ \\
& \left\langle g_{n} g_{m}\left|\hat{V}_{d d}(n-m)\right| g_{n} g_{m}\right\rangle-2\left\langle e_{n} g_{m}\left|\hat{V}_{d d}(n-m)\right| e_{n} g_{m}\right\rangle .
\end{aligned}
$$

Finally, $\hat{H}_{\mathrm{n}-\mathrm{c}}$ contains terms that do not conserve the number of molecular excitations in the system:

$$
\begin{gathered}
\hat{H}_{n-c}=\sum_{n, m}^{\prime}\left\langle e_{n} e_{m}\left|\hat{V}_{d d}(n-m)\right| e_{n} g_{m}\right\rangle\left(\hat{P}_{n}+\hat{P}_{n}^{\dagger}\right)+ \\
\frac{1}{2} \sum_{n, m}^{\prime}\left\langle e_{n} e_{m}\left|\hat{V}_{d d}(n-m)\right| g_{n} g_{m}\right\rangle\left(\hat{P}_{n} \hat{P}_{m}+\hat{P}_{n}^{\dagger} \hat{P}_{m}^{\dagger}\right)+ \\
+\sum_{n, m}^{\prime}\left[\left\langle g_{n} g_{m}\left|\hat{V}_{d d}(n-m)\right| g_{n} e_{m}\right\rangle-\right. \\
\left.\left\langle e_{n} e_{m}\left|\hat{V}_{d d}(n-m)\right| e_{n} g_{m}\right\rangle\right]\left(\hat{P}_{n}^{\dagger} \hat{P}_{n} \hat{P}_{m}+\hat{P}_{n}^{\dagger} \hat{P}_{m}^{\dagger} \hat{P}_{n}\right) .
\end{gathered}
$$


If $|g\rangle$ and $|e\rangle$ are states of well-defined parity (such as, for example, the rotational states at zero electric field), the matrix elements $D(n-m)$ as well as the linear and cubic terms in Eq. (18) must vanish. This is the case for molecular solids, such as anthracene and naphtalene, which are often considered as prototype systems for (electronic) Frenkel excitons 2. For an ensemble of molecules in an optical lattice, the magnitude of these terms can be tuned by applying an external electric field, which breaks the inversion symmetry (parity) of the molecules. To our knowledge, the role of the odd- $\hat{P}$ terms in Eq. (18) has not been explicitly studied and remains an interesting open problem. The possibility to tune these terms by varying the field dressing of the states $|g\rangle$ and $|e\rangle$ may lead to new interesting phenomena. In what follows, we assume that the effect of $\hat{H}_{\mathrm{n}-\mathrm{c}}$ is small and neglect this term.

Due to the translational invariance of the optical lattice, the Hamiltonian $\hat{H}_{\text {exc }}$ can be diagonalized by the transformation:

$$
\begin{gathered}
\hat{P}_{n}^{\dagger}=\frac{1}{\sqrt{\mathcal{N}}} \sum_{q} e^{-i q n} \hat{P}^{\dagger}(q), \\
\hat{P}_{n}=\frac{1}{\sqrt{\mathcal{N}}} \sum_{q} e^{i q n} \hat{P}(q),
\end{gathered}
$$

to yield

$$
\hat{H}_{\mathrm{exc}}=\sum_{q} E(q) \hat{P}^{\dagger}(q) \hat{P}(q)
$$

where $\hat{P}^{\dagger}(q)$ and $\hat{P}(q)$ describe the Bloch plane waves, $q$ is the (linear) momentum, and $E(q)=E_{0}+\sum_{n} J(n) e^{i q n}$ is the exciton energy forming a quasi-continuous band. For $\mathcal{N} \gg 1$, we can consider the wave vector $q$ and the energy $E(q)$ to be continuous variables.

In the nearest neighbor approximation,

$$
E_{\mathrm{NNA}}(q)=E_{0}+2 J \cos a q
$$

and

$$
J=\left\langle e_{n} g_{n+1}\left|\hat{V}_{d d}(a)\right| g_{n} e_{n+1}\right\rangle,
$$

where $a$ is the lattice constant. The energies are shown in Figure 1 for different angles $\theta$ between the one-dimensional molecular ensemble and the electric field $\mathcal{E}_{f}$ (which determines the sign of $J$ and, consequently, the shape of the dispersion curve). In the nearest neighbor approximation, $\Delta=4 J$ is the exciton bandwidth. The magnitude of $J$ is a quantitative measure of the collective excitation effects (in particular, $h / J$ is the timescale of the excitation transfer between molecules in adjacent lattice sites). Note that $J=0$ at $\theta=\theta^{*} \equiv \arccos (1 / \sqrt{3})$ : at this angle the dipole-dipole interaction vanishes, and (in the dipole approximation) the molecules become decoupled. When this happens, no excitation transfer dynamics can occur. For polar molecules with permanent dipole moments of a few

\footnotetext{
${ }^{2}$ The terms of the kind $\hat{P} \hat{P}$ and $\hat{P}^{\dagger} \hat{P}^{\dagger}$, which do not conserve the number of particles, do not vanish in natural molecular crystals with the inversion symmetry. The usual model neglecting these terms is known as the Heitler-London approximation [53. These terms can be accounted for as corrections, whose magnitude depends on the ratio between the dipole-dipole interaction matrix element $J(a)$ and the excitation energy of the molecules $E_{0}$, as well as on the structure of the molecules [61.
} 
Debye, the values of $J$ can be tens of $\mathrm{kHz}$ (for LiCs on an optical lattice with $a=400 \mathrm{~nm}$, $J \sim 25 \mathrm{kHz}$ ).

For small $q$, where the dispersion is parabolic, we can introduce the effective mass $m_{\text {eff }}=$ $-\hbar^{2} / J a^{2}$. For $\theta>\theta^{*}\left(\theta<\theta^{*}\right)$ the constant $J$ is positive (negative), and, consequently, the effective mass is negative (positive). Quasiparticles with negative effective mass have counterintuitive propagation properties: they move in the direction opposite to their wave vector (negative refraction [62]). Thus, by changing the angle $\theta$ it may be possible to tune the excitons from the regime of normal propagation to the regime of negative refraction.
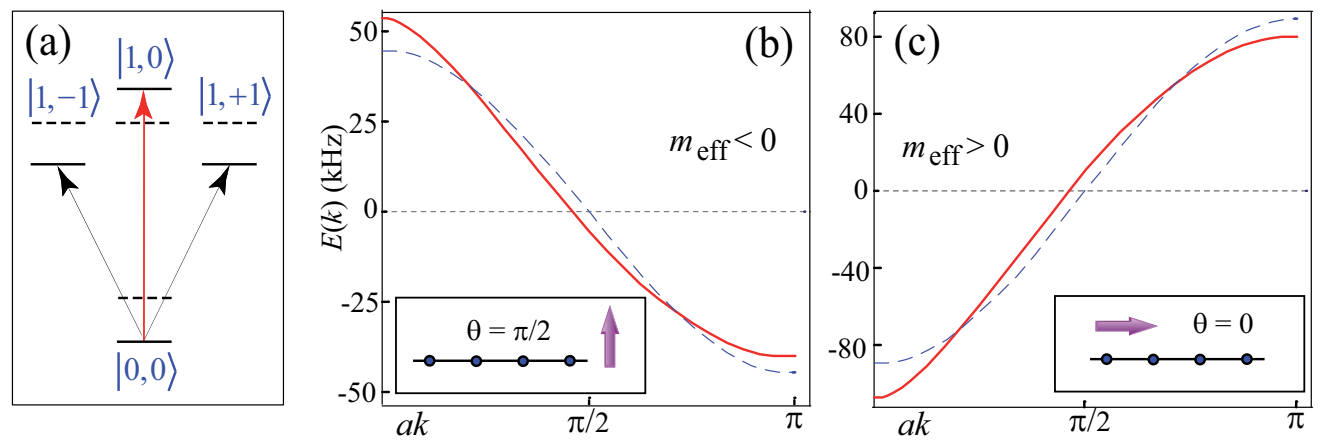

Figure 1: Panel (a): Rotational energy levels of a ${ }^{1} \Sigma$ molecule in the presence of an electric field. The dashed lines show the positions of the field-free states. Panels (b) and (c): Energy of rotational excitons in a one-dimensional array of LiCs molecules trapped on an optical lattice with the lattice constant $a=400 \mathrm{~nm}$ in the presence of an electric field of $1 \mathrm{kV} / \mathrm{cm}$. Full curves - exact numerical calculation; dashed curves - analytical result of Eq. (21). The electric field is directed perpendicular to the array axis (panel b) and parallel to the array axis (panel c).

The possibility of tuning the parameters of the exciton Hamiltonians (14), (16) and (18) in an ensemble of polar molecules on an optical lattice opens the possibility to access new regimes of Frenkel exciton physics that cannot be observed in solid state crystals. Here, we discuss a few examples that we presented in our recent papers [59], [63], [64].

\section{Nonlinear interactions of Frenkel excitons}

In this section we study non-linear interactions between rotational Frenkel excitons and show that they can be dynamically tuned by an external electric field. This is important for applications of excitons in quantum information processing [66], where excitons can be used as qubits. In particular, we consider the interplay of the dynamical interactions arising from Eq. (16) and the kinematic interaction arising from the hard-core boson nature of the exciton operators and show that the relative importance of these two interactions can be tuned.

\subsection{Dynamical and kinematic interactions}

There are two types of non-linear interactions for Frenkel excitons. The first is the dynamical interaction given by Eq. (16). This interaction is determined by the matrix elements $D(n)$ of the dipole-dipole interaction (see Eq. (17)). In the wave vector representation, the 
dynamical interaction describes wave vector conserving scattering between two excitons with the exchange of momentum $q$

$$
\hat{H}_{\text {dyn }}=\frac{1}{\mathcal{N}} \sum_{k_{1}, k_{2}, q} D(q) \hat{P}^{\dagger}\left(k_{1}+q\right) \hat{P}^{\dagger}\left(k_{2}-q\right) \hat{P}\left(k_{1}\right) \hat{P}\left(k_{2}\right) .
$$

where $D(q)=\sum_{n=1}^{\mathcal{N}} D(n) e^{-i q n}$.

The second interaction mechanism originates from the hard-core repulsion of molecular excitations. Since excitons are paulions, or hard-core bosons with long-range interactions, they effectively repel each other when placed in the same lattice site. As a result, the direct products of $\hat{P}(k)$-operators are not the eigenstates of the exciton Hamiltonian $\hat{H}_{\text {exc }}$ (14), as they would be for bosons or fermions. The commutation relations for paulions (7) yield for a two-exciton state $\left|\Phi\left(k_{1}, k_{2}\right)\right\rangle=\left|\hat{P}^{\dagger}\left(k_{1}\right) \hat{P}^{\dagger}\left(k_{2}\right)\right\rangle$ the following equation:

$$
\begin{gathered}
\hat{H}_{\mathrm{exc}}\left|\Phi\left(k_{1}, k_{2}\right)\right\rangle=\left[E\left(k_{1}\right)+E\left(k_{2}\right)\right]\left|\Phi\left(k_{1}, k_{2}\right)\right\rangle+ \\
+\frac{1}{\mathcal{N}} \sum_{\substack{q_{1}, q_{2} \\
q_{1}+q_{2}=k_{1}+k_{2}}}\left[E\left(q_{1}\right)+E\left(q_{2}\right)\right]\left|\Phi\left(q_{1}, q_{2}\right)\right\rangle .
\end{gathered}
$$

The second term on the right-hand side may be interpreted as total wave vector conserving scattering between plane-wave-like one-exciton states $\left|\hat{P}^{\dagger}\left(q_{i}\right)\right\rangle$ [52]. This type of scattering results from the kinematic interaction 67. This interaction can also be included as a perturbative term $\hat{H}_{\text {kin }}$ in the total Hamiltonian for the effective bosons introduced via the Agranovich-Toshich transformation (section 2.2). The non-linear properties of excitons (in the two-body interaction approximation) are determined by the balance between the dynamical and kinematic interactions.

\subsection{External field control of exciton-exciton interactions}

In the presence of an external electric field and with account of the dynamical interaction, the rotational Frenkel excitons are described by the Hamiltonian $\hat{H}_{\mathrm{nl}}=\hat{H}_{\text {exc }}+\hat{H}_{\text {dyn }}$ given in Eqs. (14) and (16). In the nearest neighbor approximation $\hat{H}_{\text {exc }}$ and $\hat{H}_{\text {dyn }}$ are parametrized by two constants: $D=D(a)$ and $J=J(a)$. Since $J$ determines the propagation properties of excitons, it is implicitly related to the strength of the exciton-exciton kinematic interaction: roughly speaking, the strength of repulsive interaction (scattering) between two excitons at the same lattice site is proportional to $|J|$. The constant $D$ describes the dynamical interaction. The paulionic corrections do not affect $\hat{H}_{\text {dyn }}$ so one can use the bosonic commutation relations for the $\hat{P}$-operators in this term, both in the site and wave vector representations.

Both $D$ and $J$ can be controlled by varying the magnitude and orientation of the electric field (see Figure 2). Panel (a) of Figure 2 shows the dependence of $D$ and $J$ for a onedimensional array of LiCs molecules on the field magnitude at a fixed angle between the array axis and the direction of the electric field. Note that $D$ vanishes at zero electric field (see Eq. (17)). For $\mathcal{E}_{f}=0$, the field-dressed states $|g\rangle$ and $|e\rangle$ reduce adiabatically to the pure rotational states: $|g\rangle \rightarrow\left|N=0, M_{N}=0\right\rangle$ and $|e\rangle \rightarrow\left|N=1, M_{N}=0\right\rangle$, and the matrix elements determining $D$ vanish according to the selection rules. Panel (b) shows 

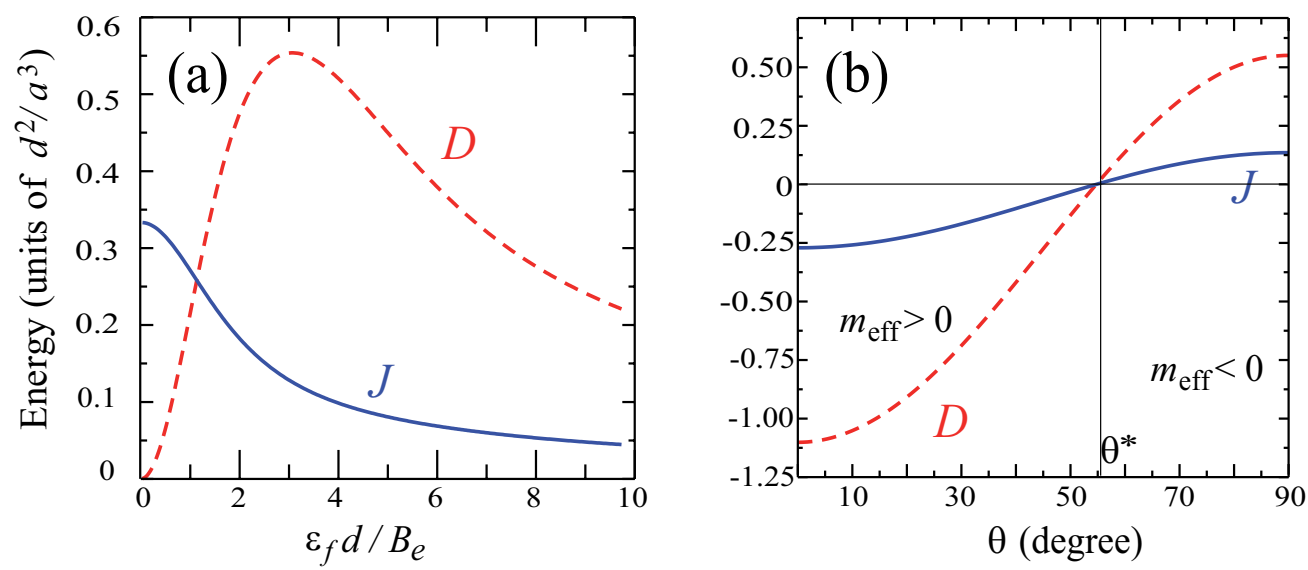

Figure 2: (a) $D$ and $J$ as functions of electric field magnitude at a fixed angle $\theta=90^{\circ}$. (b) $D$ and $J$ as functions of $\theta$ at a fixed electric field $\mathcal{E}_{f}=6 \mathrm{kV} / \mathrm{cm}$. The calculations are for a $1 \mathrm{D}$ ensemble of LiCs molecules separated by $a=400 \mathrm{~nm} ; d^{2} / a^{3}=72 \mathrm{kHz}$.

the dependence of $D$ and $J$ on the angle $\theta$ for a fixed field magnitude. Note that both $D$ and $J$ vanish at $\theta^{*}=\arccos (1 / \sqrt{3})$.

Figure 2b shows that the matrix elements $D$ and $J$ have the same sign, independent of $\theta$. As discussed in section 3, the sign of the effective mass is always opposite to the sign of $J$, and, consequently, the sign of $D$. The constant $D$ determines the dynamical interaction potential (16). Due to the linearity of the Schrödinger equation, a positive potential is attractive for a particle with negative mass and a negative potential is attractive for a particle with positive mass [59]. Therefore, the dynamical interaction in this system is always attractive [64]. The kinematic interaction is always repulsive because it arises as a consequence of the hard-core boson nature of the excitation operators.

Figure 2 shows that the values of $D$ and $J$ can be tuned in a wide range of magnitudes. For the chosen example of LiCs molecules on an optical lattice with $a=400 \mathrm{~nm}, D$ can be varied in the interval from $-80 \mathrm{kHz}$ to $40 \mathrm{kHz}$ and $J$ from $-50 \mathrm{kHz}$ to $25 \mathrm{kHz}$. Their relative magnitudes determine the relative contributions of the kinematic and dynamical interactions. By changing the ratio between $D$ and $J$, one can explore the interaction regimes dominated by the different interactions. In particular, prevailing attraction may result in the formation of bound two-exciton complexes known as biexcitons [64]. They form in the low dimensional systems when $|D|>2|J|$ [65]. In 1D they appear as a single state band split form the continuum of the two-particle states. As a consequence of the correlation between the signs of $D$ and the effective mass, the biexciton state appears below (above) the two-particle continuum for negative (positive) $D$, and can be continuously tuned between these two positions by varying the angle between the electric field and the intermolecular axis. The properties of biexcitons can be engineered by varying the ratio $D / 2 J$ by tuning the magnitude of the electric field. In particular, for an ensemble of LiCs molecules, the biexciton begins to appear at $\mathcal{E}_{f} \approx 3.6 \mathrm{kV} / \mathrm{cm}$, when $D=2 J$. With the increase of the electric field its binding energy (and, correspondingly, its splitting from the continuum states) increases, and the wave function shrinks, so that at $D \gg 2 J$ a biexciton is a strongly correlated state of two molecular excitations separated by one or two lattice constants. The formation of a biexciton thus resembles the association of a molecule by combining two atoms. 
When $D=0$, the dynamical interaction vanishes, and the kinematic interaction dominates. It should be mentioned that the effect of the kinematic interactions on Frenkel exciton dynamics has not yet been observed in experiments. To this end, it would be useful to find a mechanism for tuning the kinematic interaction as well. We explore this in the following section.

\subsection{Suppression of kinematic interaction}

Although the kinematic interaction is inherent to molecular crystals as an intrinsic consequence of the exciton operator statistics, it may be possible to generate excitations that do not experience kinematic interactions. To find the conditions for such excitations, we neglect the dynamical interactions and look for solutions of the two-particle Schrödinger equation

$$
\hat{H}_{\mathrm{exc}}\left|\Psi_{K}\right\rangle=E_{K}\left|\Psi_{K}\right\rangle
$$

in the form

$$
\left|\Psi_{K}\right\rangle=\sum_{k} C_{K}(k) \hat{P}^{\dagger}(K / 2+k) \hat{P}^{\dagger}(K / 2-k)|\mathrm{vac}\rangle
$$

where $k=\left(k_{1}-k_{2}\right) / 2$ is the relative wave vector of two interacting excitons, and $K=$ $k_{1}+k_{2}$. The expansion coefficients in Eq. (26) satisfy the following equation

$$
\left(\varepsilon_{K}(k)-E_{K}\right) C_{K}(k)=\frac{\varepsilon_{K}(k)}{\mathcal{N}} \sum_{k^{\prime}} C_{K}\left(k^{\prime}\right),
$$

where $\varepsilon_{K}(k)=E(K / 2-k)+E(K / 2+k)$ is the total energy of two interacting excitons. The two-particle amplitude in the site representation, which depends on the relative distance $r=n-m$ between two molecular excitations, is $C_{K}(r)=1 / \mathcal{N} \sum_{k} e^{i k r} C_{K}(k)$. Since $C_{K}(r=$ $0)=0$, the right-hand side of Eq. (27) vanishes. Therefore, Eq. (27) can be satisfied only if

$$
\varepsilon_{K}(k)=\text { const }=E_{K} .
$$

The corresponding wave function is $(\mathcal{N}-1)$-time degenerate ( $n$-degeneracy):

$$
\left|\Psi_{K}^{(n)}\right\rangle=\frac{1}{\sqrt{\mathcal{N}}} \sum_{k} B_{K}(n) e^{i k n} \hat{P}^{\dagger}(K / 2+k) \hat{P}^{\dagger}(K / 2-k)|\mathrm{vac}\rangle
$$

where the quantum number $n$ determines the fixed distance $r=n a$ between the excited molecules: $n=1,2, \ldots, \mathcal{N}-1$. These states describe two correlated excitations that do not experience the kinematic interaction.

The key requirement (28) - the $k$-independence of the two-particle energy $\varepsilon_{K}(k)-$ is however not easy to satisfy. One possibility is to consider a specific choice of $K$. In the nearest neighbor approximation, when $E(q)=E_{0}+2 J \cos a q$, the two-particle energy written in terms of $K$ and $k$ is $\varepsilon_{K}(k)=2 E_{0}+4 J \cos (a k) \cos (a K / 2)$. It reduces to a constant if $K=\pi / a$. Thus, with the accuracy up to corrections coming from the interactions beyond the nearest neighbors and quadrupole interactions, such a pair immune to the kinematic interaction can be produced starting from a pair of excitons with $q_{1}+q_{2}=\pi / a$. 
Combined with the possibility to control the dynamical interaction strength by the electric field magnitude, this provides a system in which both attractive and repulsive interactions between quasiparticles can be independently tuned.

Another possibility to produce a two-particle state with the energy independent of $k$ can be realized using the three-level structure of the first rotationally excited state of ${ }^{1} \Sigma$ molecules (see Figure 1a). At low electric fields, when the energy separation between the levels $|N=1, M=0\rangle$ and $|N=1, M= \pm 1\rangle$ is small, the different excitation branches may be mixed, which corresponds to configuration mixing in solid state molecular crystals [53]. Due to the symmetry properties of the molecular system considered here, the state with $M=0$ is decoupled from the states with $M= \pm 1$ if the electric field is parallel or perpendicular to the molecular ensemble. For other angles $\theta$, the collective excitations give rise to three exciton modes which we denote $\alpha, \beta$ and $\gamma$, containing contributions from all three molecular transitions [59]:

$$
\hat{P}_{\rho=\alpha, \beta, \gamma}^{\dagger}(k)=\sum_{M=-1,0,1} u_{M}^{\rho}(k) \hat{P}_{M}^{\dagger}(k),
$$

where $\hat{P}_{M}^{\dagger}(k)=(1 / \sqrt{\mathcal{N}}) \sum_{n} e^{i k n} \hat{P}_{n, M}^{\dagger}$ are the exciton operators corresponding to the molecular transition to the excited state with projection $M=M_{N}$. Operators (30) diagonalize the four-level excitonic Hamiltonian yielding

$$
\hat{H}_{\mathrm{exc}}^{\{M\}}=\sum_{k ; \rho=\alpha, \beta, \gamma} E_{\rho}(k) P_{\rho}^{\dagger}(k) P_{\rho}(k) .
$$

At high electric field, the $\gamma$-mode is split from the other two modes, leading to the isolated one-band exciton discussed throughout this article. However, the other two branches correspond to the excitations of the degenerate molecular states and remain mixed. Their $k=0$ state can be accessed by microwave field with circular polarization. The non-zero $k$ states can be probed by Raman transitions combining photons with linear and circular polarization. In the limit of high electric fields, the energies of these excitons can be written as

$$
\begin{gathered}
E_{\alpha}(k ; \theta)=E_{1}\left(\mathcal{E}_{f}\right)+2\left|\alpha_{N=0}\left(\mathcal{E}_{f}\right)\right|^{2} j(k)\left(\cos ^{2} \theta-2 / 3\right), \\
E_{\beta}(k)=E_{1}\left(\mathcal{E}_{f}\right)+2\left|\alpha_{N=0}\left(\mathcal{E}_{f}\right)\right|^{2} j(k) / 3,
\end{gathered}
$$

where $E_{1}\left(\mathcal{E}_{f}\right)$ is the transition energy between the field-dressed ground state and the fielddressed excited state with $M= \pm 1 ; j(k)=\left(d^{2} / a^{3}\right) \sum_{n=1}^{\mathcal{N} / 2} \cos (a k m) / m^{3}$, and $\alpha_{N}\left(\mathcal{E}_{f}\right)$ are defined in Eq. (12). The kinematic interaction in the presence of several branches is more complicated, but it can be shown that the condition (28) also applies to the multi-branch problems. It can be seen that, at a particular angle $\theta=\arccos \sqrt{1 / 3}$,

$$
E_{\alpha}(k ; \theta)+E_{\beta}(k)=2 E_{1}\left(\mathcal{E}_{f}\right)=\text { const, }
$$

so the kinematic interaction must be absent. Eq. (33) is satisfied for arbitrary values of $K$. We note that for the $\gamma$-branch, the matrix elements of the dipole-dipole interaction vanish at $\theta=\theta^{*}$ so the $\gamma$-state becomes dispersion-less. However, the matrix elements giving rise to the other two exciton states remain non-zero at this angle, see Eqs. (32). It is the cancellation of the dispersions of $E_{\alpha}(k)$ and $E_{\beta}(k)$ that leads to the suppression of the kinematic interaction. 


\section{Rotational excitons in a tunable disordered poten- tial}

Dynamics of quantum particles in disordered potential has been extensively studied in relation to Anderson localization [68], propagation of light [69] and particles [70] through disordered media, phase transitions between insulating and conducting states [71], to name a few examples. As a result of these studies, many properties of disordered systems, such as the role of the density of states or the correlation functions are well understood. At the same time, there are still many problems that remain open. A few examples include the role of disorder in the transition to the glassy state [72], transition from a superconductor to insulator with increasing disorder [73], and the counterintuitive behavior of conductivity in some disordered quasi-crystals [74].

In this section we show that ultracold molecules trapped on an optical lattice offer the possibility to study quasiparticles in the presence of dynamically tunable disordered potential. We do not consider the effects of unavoidable natural disorder, such as unoccupied lattice sites, lattice potential inhomogeneity or fluctuations of the electric field. Always present in experiments with atoms and molecules on optical lattices, the natural disorder induces localization and decoherence of excitons leading to homogeneous broadening of exciton dispersion curves. We assume that the effects of natural disorder can be reduced to a small fraction of the exciton bandwidth. Here, we consider the possibility of applying an external disordered potential that could be varied to allow the observation of real-time dynamics of disorder-induced phenomena.

\subsection{Optical lattice with tunable disorder}

There are several different models of disorder [75], and some of them can potentially be realized in an optical lattice with ultracold molecules. First, suppose that a small fraction of molecules trapped on the lattice (host molecules) is replaced with molecules of different kind (impurities). This results in substitutional disorder. We assume that all impurity molecules are identical. The impurities break the translational symmetry of the system and therefore scatter excitons. This scattering is elastic and modifies the direction of exciton wave propagation, but not the absolute value of the exciton wave vectors.

An impurity introduced into the molecular crystal in general differs from the host molecules by the molecular transition energy, $E_{i}$, and by the dipole moment, $d_{i}$, which modifies the dipole-dipole coupling strength. In the presence of a single impurity at the lattice site $n=0$, we can write the total Hamiltonian of the system as

$$
\hat{H}_{\text {tot }}=\hat{H}_{\mathrm{exc}}+V_{0} \hat{P}_{0}^{\dagger} \hat{P}_{0}+\sum_{n \neq 0} \Delta J(n)\left(\hat{P}_{n}^{\dagger} \hat{P}_{0}+\hat{P}_{0}^{\dagger} \hat{P}_{n}\right)
$$

where $V_{0}=E_{i}-E_{0}$ and $\Delta J(r)$ is the difference between the host-host and host-impurity excitation transfer constants. The exciton-impurity interaction can thus be described as a sum of a delta-function potential with strength $V_{0}$ and a perturbation due to the difference in the dipole moments of the host and impurity molecules. If the matrix of the operator (34) is evaluated in the basis of exciton states in the site representation, one finds that $V_{0}$ perturbs the diagonal matrix elements and $\Delta J$ the off-diagonal matrix elements. Thus, the constants $V_{0}$ and $\Delta J(r)$ give rise to diagonal and off-diagonal disorder. 
For a properly chosen mixture of diatomic molecules, the magnitude and the sign of $V_{0}$ can be tuned by an external electric field. For example, Figure 3 shows that this can be achieved in an array of LiCs molecules doped with LiRb molecules. Generally, it should be possible to tune $V_{0}$ from a negative value to a positive value in a mixture of ${ }^{1} \Sigma$ diatomic molecules $A B$ and $C D$, when the dipole moment of $A B$ is greater and the rotational constant of $A B$ is smaller.

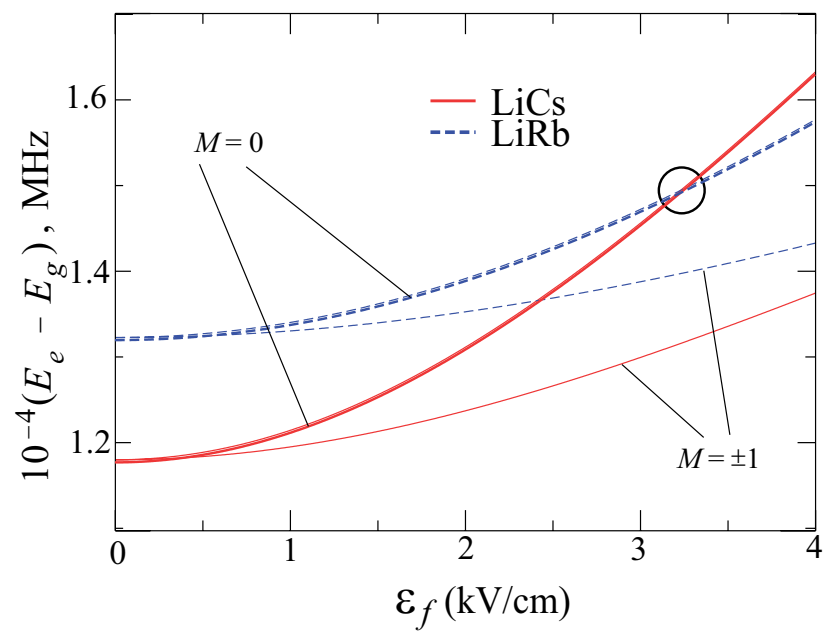

Figure 3: Excitation energies of non-interacting molecules in an electric field for transitions $|N=0, M=0\rangle \rightarrow|N=1, M\rangle$ with $M=0$ (upper curve) and $M= \pm 1$ (lower curve) vs electric field for LiCs and LiRb.

It may also be possible to realize a system with tunable diagonal disorder in a singlespecies ensemble of molecules by applying intense laser beams focused on a small part of the lattice. Molecules at the focus of the beam must experience larger ac Stark shifts than the molecules outside the beam focus. These molecules are equivalent to impurities because their energy level structure is different. The degree of detuning $V_{0}$ can be adjusted by varying the laser field strength. For small fields, it may be possible to neglect the offdiagonal disorder terms, which suggests a unique possibility to differentiate between the effects of diagonal and off-diagonal disorder.

\subsection{Resonant enhancement of exciton-impurity scattering}

The properties of waves with arbitrary dispersion in the presence of local defects have been studied in Ref. [76]. Similar considerations were later applied to excitons in a crystal with substitutional disorder in Ref. [77], where the exciton-impurity scattering cross section, which characterizes their interaction, was derived. The exciton-impurity interactions lead to the appearance of bound exciton states, which can capture excitons in solid state crystals [78. The constants $V_{0}$ and $\Delta J$ in Eq. (34) determine the character of the exciton-impurity interactions in natural solids.

We first consider an ensemble of molecules driven by several focused laser beams with the same strength, so that $\Delta J=0$, and all impurities are characterized by the same value of $V_{0}$. This system may allow for the possibility to explore the dependence of excitonimpurity scattering cross sections not only on the exciton wave vector $k$, but also on the impurity scattering strength $V_{0}$, which is not possible in conventional solids. In particular, 
as we show below, the scattering cross section can be resonantly enhanced, if the potential produces a shallow bound state at small values of $V_{0}$ [59].

For a particle with the parabolic dispersion $E=\hbar^{2} q^{2} / 2 m_{*}$ in a $d$-dimensional deltalike potential $\hat{H}_{\delta}=a^{d} V_{0} \delta(\mathbf{r})$ the bound state is split from the continuum states by the energy $E_{b}$, which depends on the dimensionality of the system. The binding energy can be obtained from the following equations:

$$
\begin{aligned}
& \sqrt{\frac{E_{b}^{(3 \mathrm{D})}}{E_{\mathrm{loc}}}} \arctan \sqrt{\frac{E_{\mathrm{loc}}}{E_{b}^{(3 D)}}}=1+\operatorname{sgn}\left(m_{*}\right) \frac{2 E_{\mathrm{loc}}}{\pi V_{0}} \quad \text { for } 3 \mathrm{D} \\
& \frac{E_{b}^{(2 \mathrm{D})}}{E_{\mathrm{loc}}}=\left[\exp \left(-\operatorname{sgn}\left(m_{*}\right) \frac{4 E_{\mathrm{loc}}}{\pi V_{0}}\right)-1\right]^{-1} \quad \text { for } 2 \mathrm{D} \\
& \sqrt{\frac{E_{b}^{(1 \mathrm{D})}}{E_{\mathrm{loc}}}}=-\frac{\operatorname{sgn}\left(m_{*}\right) V_{0}}{E_{\mathrm{loc}}} \arctan \sqrt{\frac{E_{\mathrm{loc}}}{E_{b}^{(1 D)}}} \text { for } 1 \mathrm{D}
\end{aligned}
$$

where $E_{\mathrm{loc}}=\hbar^{2} \pi^{2} / 2\left|m_{*}\right| a^{2}$ is the localization energy of a particle with the mass $\left|m_{*}\right|$ in a region of dimension $a$. Note that these equations have solutions only for negative (positive) $V_{0}$ and positive (negative) mass $m_{*}$.

Figure 4 a shows the behavior of the local states as functions of the dimensionless potential strength $\left|V_{0}\right| / E_{\text {loc }}$. In $1 \mathrm{D}$ and $2 \mathrm{D}$, an attractive delta-like potential always produces a bound state, and in 3D only starting from a finite value: $\left|V_{0}\right|>2 E_{\mathrm{loc}} / \pi$. Resonant scattering may play an important role if the resonant enhancement of the scattering cross section at $E_{b} \rightarrow 0$ is reached at a finite value of $V_{0}$. For instance, in $1 \mathrm{D} E_{b}^{(1 D)} \approx \pi^{2} V_{0}^{2} / 4 E_{\mathrm{loc}}$ at vanishing $V_{0}$, and the shallowing of the bound state is accompanied by the vanishing of the scattering potential itself. In turn, in $3 \mathrm{D}$ one can expect a resonant enhancement of the scattering cross section for the potential $\left|V_{0}\right| \sim 2 E_{\mathrm{loc}} / \pi=\pi \hbar^{2} /\left|m_{*}\right| a^{2}$. Near this potential strength, $E_{b}^{(3 D)} \approx E_{\mathrm{loc}}\left(2 / \pi+V_{0} \operatorname{sgn}\left(m_{*}\right) / E_{\mathrm{loc}}\right)^{2}$. In $2 \mathrm{D}$, any potential $\left|V_{0}\right|<0.2 E_{\mathrm{loc}}$ produces a shallow bound state, whose energy tends exponentially to zero with vanishing impurity potential strength: $E_{b}^{(2 \mathrm{D})} \approx E_{\mathrm{loc}} \exp \left[4 E_{\mathrm{loc}} / \pi V_{0} \operatorname{sgn}\left(m_{*}\right)\right]$ (we recall that $V_{0}$ and $m_{*}$ should have different signs for a bound state to appear). This exponential dependence leads to efficient resonant scattering for all $0<V_{0}<0.2 E_{\mathrm{loc}}$ : as the potential strength in $2 \mathrm{D}$ exponentially exceeds the kinetic energy of the scattered wave, then, in contrast to the 1D case, the resonant scattering cannot be considered as weak, even at vanishingly small $V_{0}$. At the same time, this means that for $\left|V_{0}\right| \ll 0.2 E_{\text {loc }}$ the local state is so close to zero, that the potential scatters resonantly only excitons with $k=0$, which in fact do not propagate.

For small wave vector excitons, $E(k)=\left(E_{0}+2 J\right)-J a^{2} k^{2}$, so that $\hbar^{2} / m_{*}=-2 J a^{2}$, and $E_{\text {loc }}=\pi^{2} J$. Borrowing the results from Ref.[79], we write the scattering cross sections in $2 \mathrm{D}$ and $3 \mathrm{D}$ for $a k \ll 1$ as explicit functions of the potential strength $V_{0}$, and express them as functions of the bound state energy $E_{b}$ and the kinetic energy $T(k)=\hbar^{2} k^{2} / 2\left|m_{*}\right|=|J|(a k)^{2}$ (these expressions correspond to the limit when both $T(k)$ and $E_{b}$ are much smaller than 

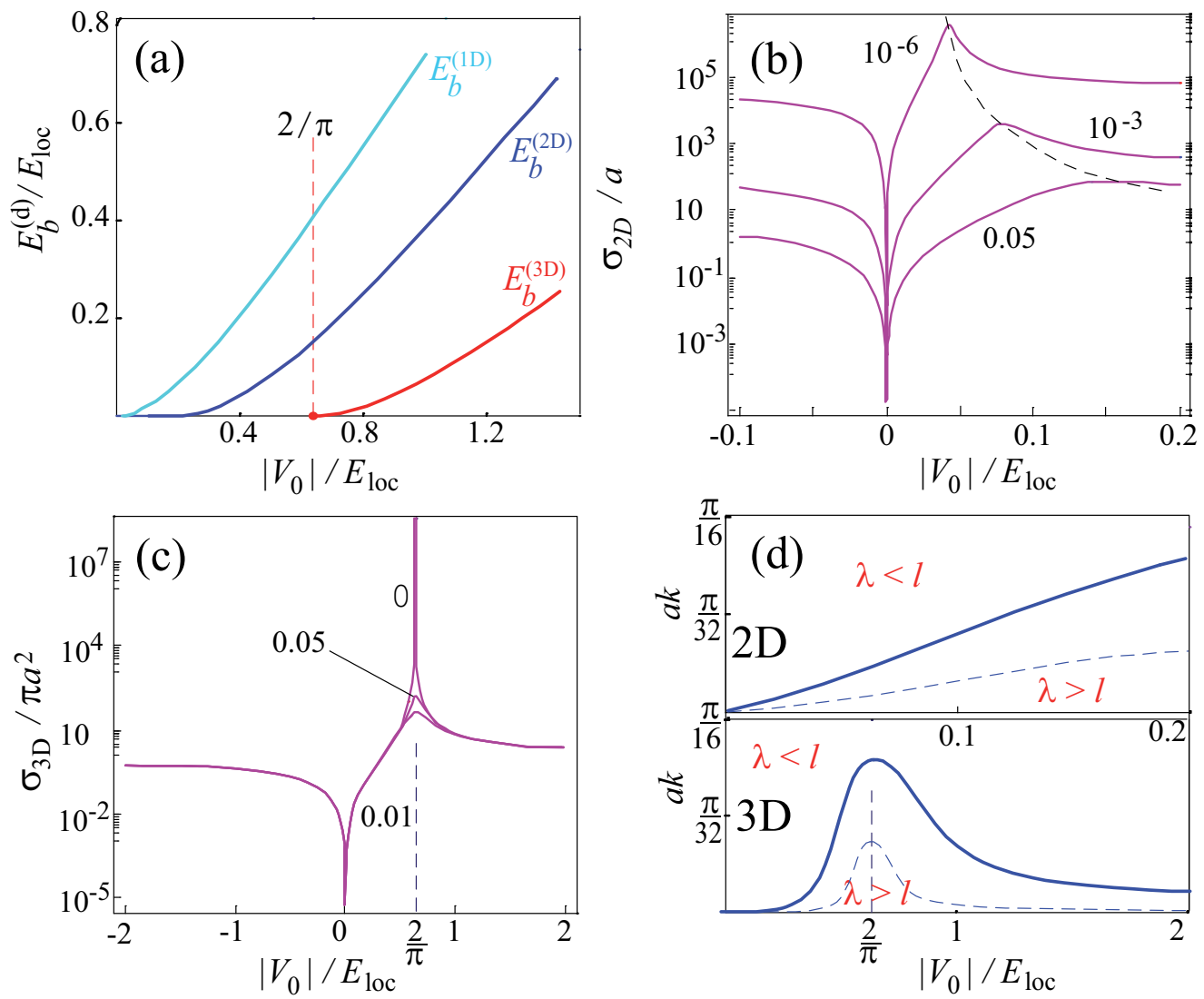

Figure 4: (a) Dependence of the bound state energy $E_{b}^{(d)}$ on potential strength $V_{0}$ in different dimensions. (b,c) The exciton-impurity scattering cross sections for 2D (b) and 3D (c) as functions of the potential strength for different values of $a k$ (shown near each line). (d) Different propagation regimes in 2D (top) and 3D (bottom) for 1\% of impurities (thick solid line) and $0.1 \%$ of impurities (thin dashed line).

$\left.E_{\mathrm{loc}}\right)$ :

$$
\begin{aligned}
& \frac{\sigma_{2 \mathrm{D}}\left(k, V_{0}\right)}{a}=\frac{\pi^{2} / a k}{\frac{\pi^{2}}{4}+\left[\ln \frac{a k}{\pi}-\frac{2 E_{\mathrm{loc}}}{\pi V_{0}} \operatorname{sgn}\left(m_{*}\right)\right]^{2}}=\frac{4 \pi^{2} / a k}{\pi^{2}+\left[\ln \left(E_{b}^{(2 D)} / T(k)\right)\right]^{2}}, \\
& \frac{\sigma_{3 \mathrm{D}}\left(k, V_{0}\right)}{\pi a^{2}}=\frac{1}{\left(\frac{a k}{2}\right)^{2}+\left[\left(\frac{a k}{\pi}\right)^{2}-\frac{2 E_{\mathrm{loc}}}{\pi V_{0}} \operatorname{sgn}(m)-1\right]^{2}}=\frac{4 E_{\mathrm{loc}} / \pi^{2}}{E_{b}^{(3 \mathrm{D})}+T(k)} .
\end{aligned}
$$

Figures 4b and 4c show the scattering cross sections for 2D and 3D (note the divergence of $\sigma_{3 \mathrm{D}}$ at $\left.k \rightarrow 0, V_{0} \rightarrow 2 E_{\mathrm{loc}} / \pi\right)$. These results demonstrate that by tuning the potential strength from zero to the critical value $\left(V_{\mathrm{cr}}^{(2 \mathrm{D})} \leq 0.1 E_{\mathrm{loc}}\right.$ and $\left.V_{\mathrm{cr}}^{(3 \mathrm{D})}=2 E_{\mathrm{loc}} / \pi\right)$, one can vary the value of the scattering cross section for small wave vector excitons by many orders of magnitude.

The single-impurity scattering cross sections determine the regimes of exciton propagation in the lattice with a small admixture of impurities. According to the Ioffe-Regel criterion [80], excitons with the wavelength $\lambda=2 \pi / k$ are strongly localized when $\lambda / 2 \pi \geq l$ 
( $l$ is the elastic mean free path). When $\lambda / 2 \pi \ll l$ excitons have plane-wave-like character, though, in some cases, weak localization of excitons can be achieved [81]. Excitons propagate without scattering when $l$ is on the order of the lattice size.

For the ensemble of polar molecules trapped on an optical lattice, the elastic mean free path of excitons $l \sim 1 / \sigma c$ for a given concentration $c$ of impurities can be dynamically tuned by varying the strength of the electric field that modifies the disordered potential and, consequently, $\sigma$. This may allow a possibility to transfer excitons dynamically from the regime of ballistic propagation to the regimes of weak or strong localization. Figure 4 $\mathrm{d}$ shows the $\left(V_{0}, k\right)$-diagram of different propagation regimes for $2 \mathrm{D}$ (top) and 3D (bottom) lattices with the concentration of impurities $1 \%$. The solid lines correspond to the condition $l=\lambda / 2 \pi$. Below these lines, the exciton wave length $\lambda$ exceeds $2 \pi l$, which corresponds to the Anderson localization regime. Above the lines, the states are in general delocalized. The dashed line is plotted for the impurity concentration $0.1 \%$. The diagrams are not complete without another important parameter, the phase-breaking length $l_{\phi}$. This length accounts for inelastic scattering processes. The interference effects leading to weak and strong localization are only possible if $l \ll l_{\phi}$ [81]. The phase-breaking length should be calculated for a given realization of the experimental system with the account of the major loss channels for rotational excitons.

\subsection{Disorder correlations and localization-delocalization crossover}

Consider now a lattice with multiple impurities formed by molecules of a different type. This leads to significant values of $\Delta J \neq 0$. Quantum particles in the presence of a random distribution of scattering centers undergo coherent localization, and in 1D all states are exponentially localized even in presence of disorder of arbitrarily small magnitude [82. However, when the disorder potential exhibits short-range correlations, particular states may become delocalized [83. Delocalized states may even form a continuous band, if the correlations are long-range, so that a mobility edge exists between localized and delocalized states [84]. Studies of correlation-induced delocalization of quantum states are important for understanding quantum transport in disordered systems.

The substitutional disorder introduces both the diagonal $\left(V_{0}\right)$ and off-diagonal $(\Delta J)$ disorder. As a consequence, $V_{0}$ and $\Delta J$ are correlated, and the spectrum of a 1D disordered lattice with a two-molecule mixture must always contain one delocalized state [85]. The energy of this state is determined by the relation between $V_{0}$ and $\Delta J$. The delocalization occurs because the diagonal and off-diagonal perturbations compensate one another.

As demonstrated by Figure 3, $V_{0}$ can be tuned by shifting the rotational levels of host and impurity molecules simultaneously using a static electric field. In particular, at the field corresponding to the encircled region, $V_{0}$ vanishes, while $\Delta J$ remains finite. Tuning the electric field around this value allows for the possibility to exploit both the positive and negative values of $V_{0}$. Choosing the appropriate value of $V_{0}$ can be used to induce the delocalization of any eigenstate of the system. For states near the origin of the Brillouin zone, delocalization occurs at $V_{0} \approx-4 \Delta J$ [59]. Figure 5 shows the evolution of a particular eigenstate with $V_{0}$. At $V_{0}=0$, the state is localized due to non-zero $\Delta J$ and exhibits the characteristic exponential profile. At $V_{0}=22 \mathrm{kHz}$, the diagonal disorder compensates the effects of the off-diagonal disorder, and the state becomes delocalized. Tuning $V_{0}$ further results in the localization of this eigenstate. 

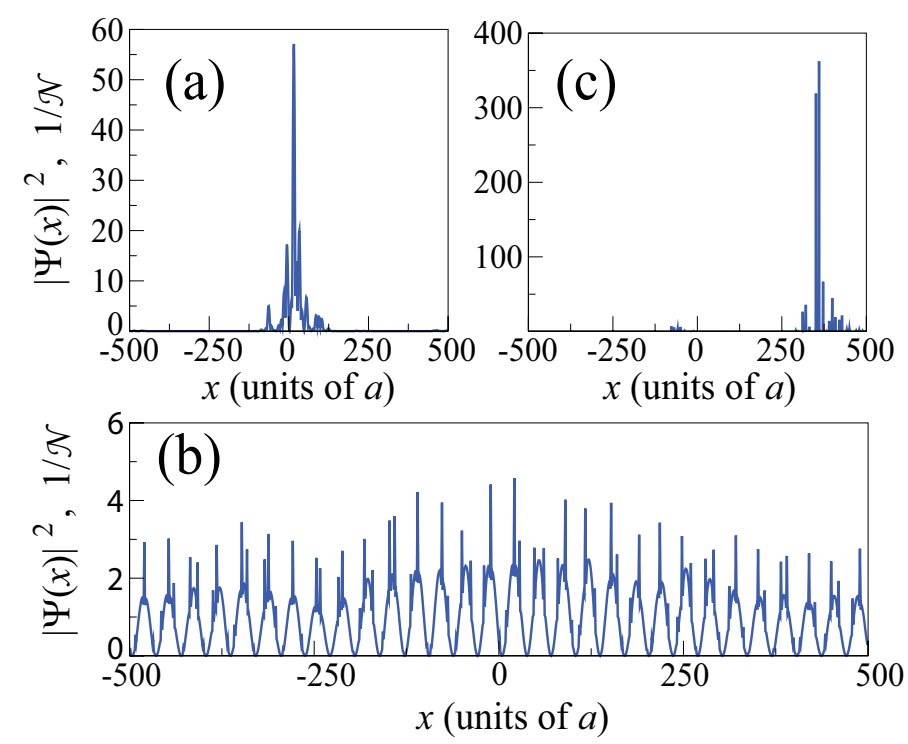

Figure 5: Probability density $|\Psi(x)|^{2}$ describing an exciton near the top of the energy spectrum for a 1D array of 1000 LiCs molecules with $10 \%$ homogeneously and randomly distributed LiRb impurities. Panels correspond to different values of $V_{0}$ : (a) $V_{0}=0$, (b) $V_{0} / h=22 \mathrm{kHz}$, and (c) $V_{0} / h=100 \mathrm{kHz}$. The difference of the dipole moments of LiCs and LiRb molecules leads to the value $\Delta J=-6.89 \mathrm{kHz}$. Figure is taken from Ref. [59].

\section{Quantum simulation of Holstein polaron model}

Rotational Frenkel excitons can be used for quantum simulation of model Hamiltonians that cannot be engineered with atoms, molecules or photons as probe particles. Here, we discuss an important example illustrating the possibility of engineering the Holstein polaron model with collective excitations of molecules on an optical lattice.

Polaron is a quasiparticle that describes an electron in a crystal lattice dressed by lattice phonons. The interaction properties of polarons are currently researched in an effort to understand the mechanism of high- $T_{\mathrm{c}}$ superconductivity [86] and quantum transport in open quantum systems [87]. Quasiparticles similar to polarons can be created by placing an impurity in a Fermi degenerate gas of ultracold atoms, as demonstrated in several recent experiments [24]. The impurity, produced by changing the internal state of one of the ultracold atoms, can be coupled to the Fermi sea via a Feshbach resonance. This gives rise to Fermi polarons. However, lattice phonons are bosons. Therefore, a better model of electrons in solid state crystals should be based on coupling a probe particle to bosons. While this may be achieved by placing an impurity in a Bose-Einstein condensate of ultracold atoms, no such experiments have been reported to date.

In a recent study [63], Herrera and Krems showed that the dipole-dipole interactions between polar molecules on an optical lattice can be exploited to engineer controllable couplings between excitons and lattice phonons. In this system, the phonons are associated with the oscillatory motion of molecules in the lattice potential

$$
\hat{V}=\sum_{n} M \omega_{0}^{2} \delta r_{n}^{2} / 2+\sum_{n, m}^{\prime} U_{g} /|n-m|^{3}
$$

where $\delta r_{n}$ is a small deviation from the equilibrium position of the molecule in site $n, M$ is the mass of the molecules, $\omega_{0}$ is the trapping frequency, and $U_{g}=R^{3}\left\langle g g\left|\hat{V}_{d d}(R)\right| g g\right\rangle$. 
The first term in Eq. (37) describes uncoupled oscillations of molecules in their respective lattice sites and depends on the intensity of the trapping laser that determines the trapping frequency $\omega_{0}$. The second term accounts for the collective motion by coupling molecules in different sites. By introducing the operators $\hat{a}_{\nu}^{\dagger}(k)$ and $\hat{a}_{\nu}(k)$ for the phonon mode $\nu$ with wave vector $k$, we can write the phonon Hamiltonian as

$$
\hat{H}_{\mathrm{ph}}=\sum_{\nu, k} \hbar \omega_{\mathrm{ph}}(k) \hat{a}_{\nu}^{\dagger}(k) \hat{a}_{\nu}(k)
$$

where

$$
\hbar \omega_{\mathrm{ph}}(k)=\omega_{0} \sqrt{1+\left(12 U_{g} / a^{5} M \omega_{0}^{2}\right) \sum_{m>0}(1-\cos m k) / m^{5}} .
$$

The phonon spectrum is gapped, as $\omega(k \rightarrow 0) \rightarrow \omega_{0}$, and resembles that of optical phonons in solid state crystals.

The exciton-phonon interaction is obtained by expanding the matrix elements of the dipole-dipole interaction in a Taylor series to yield:

$$
\begin{gathered}
\hat{H}_{\mathrm{exc}-\mathrm{ph}}=\sum_{k, \nu ; n} g_{F}^{\nu}(k)\left(\hat{a}_{\nu}^{\dagger}(k)+\hat{a}_{\nu}(k)\right) \hat{P}_{n}^{\dagger} \hat{P}_{n}+ \\
\sum_{k, \nu ; n, m}{ }^{\prime} g_{J}^{\nu}(k)\left(\hat{a}_{\nu}^{\dagger}(k)+\hat{a}_{\nu}(k)\right) \hat{P}_{n}^{\dagger} \hat{P}_{m},
\end{gathered}
$$

where $g_{F}^{\nu}(k) \approx F / a \sqrt{M \omega_{0}}, g_{J}^{\nu}(k) \approx J / a \sqrt{M \omega_{0}}, F=\left\langle e g\left|\hat{V}_{d d}(a)\right| e g\right\rangle-\left\langle g g\left|\hat{V}_{d d}(a)\right| g g\right\rangle$, and $J$ is the excitation transfer matrix element defined in Eq. (14). The first term in Eq. (40) describes the phonon-modulated transition energies, the second - the phonon-modulated excitation transfer.
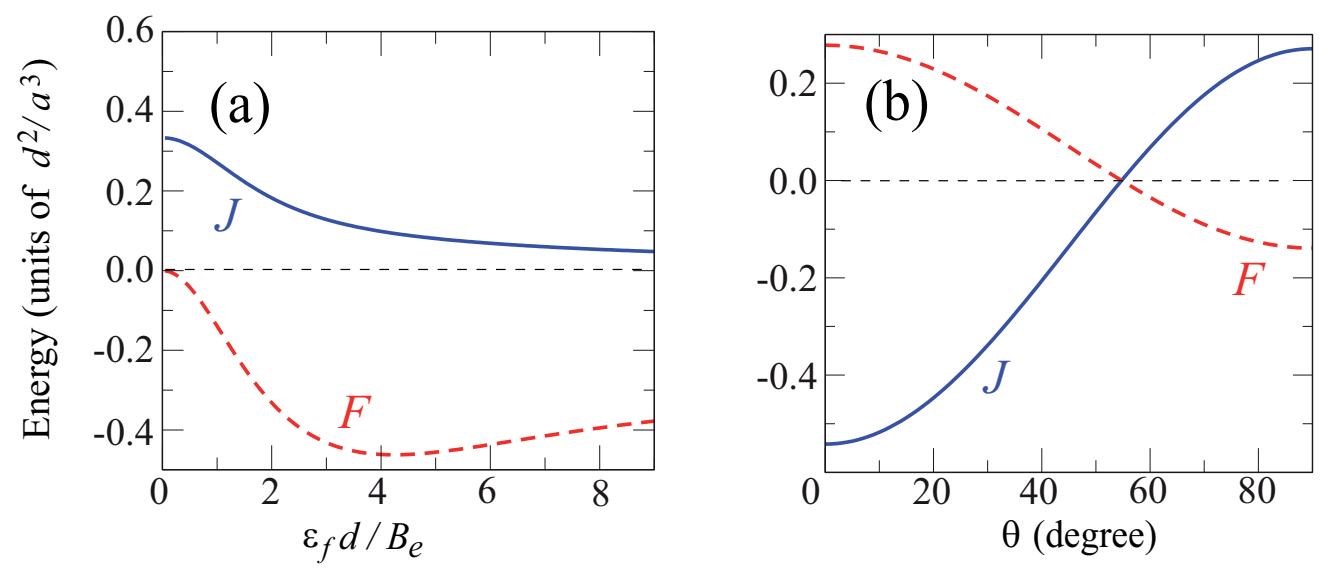

Figure 6: $F$ and $J$ as functions of the electric field magnitude at a fixed angle $\theta=90^{\circ}$ (a) and of the angle $\theta$ at $d \mathcal{E}_{f}=B_{e}$ (b). Calculations are for a 1D ensemble of LiCs molecules separated by $a=400 \mathrm{~nm} ; d^{2} / a^{3}=72 \mathrm{kHz}$. This is a modified version of the figure taken from Ref.[63].

The exciton-phonon coupling constants $g_{F}$ and $g_{J}$ depend on the molecular states $|g\rangle$ and $|e\rangle$, which can be tuned by an external electric field. The dependence of the constants 
$F$ and $J$ on the electric field magnitude $\mathcal{E}_{f}$ and direction $\theta$ is shown in Figure 6. In the limit $g_{F} \gg g_{J}$ the Hamiltonian (40) describes the Holstein polaron model [88]; this limit corresponds to large values of $\mathcal{E}_{f}$. In the opposite limit, when $g_{F} \ll g_{J}$, it corresponds to the model of particle-boson coupling by Su, Schrieffer and Heeger [89]; this is the limit of small $\mathcal{E}_{f}$.
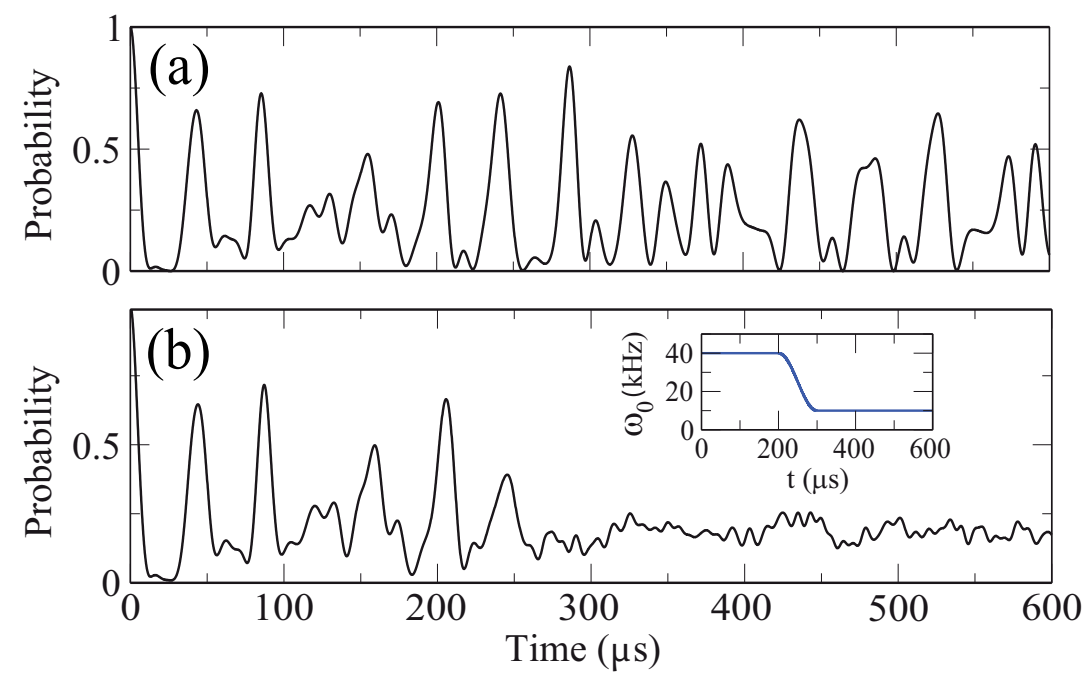

Figure 7: Excitation energy transfer in a 1D array of five LiCs molecules separated by 400 $\mathrm{nm}$ in a de electric field perpendicular to the array. (a) Evolution of the excitation probability for the first molecule of the array, when no phonons are present. The revivals occur due to the finite size of the molecular array and the reflection of the exciton wavepacket from the edge of the ensemble. (b) The same as in (a), but with phonons in an optical lattice with trapping frequency $\nu_{0}=\omega_{0} / 2 \pi$ varying in time as indicated in the inset. The field strength is $0.5 \mathrm{kV} / \mathrm{cm}$. The figure is taken from Ref. 63]

The exciton-phonon coupling for molecules in an optical lattice can also be controlled by varying the trapping frequency $\omega_{0}$, which is proportional to the intensity of the trapping laser and enters both of the coupling constants as $1 / \sqrt{\omega_{0}}$. Increasing the exciton-phonon coupling strength results in an increase of the effective mass of the polaron. In the limit of strong coupling, polarons are very massive and localized. The coherent propagation is impeded and polarons can only propagate by random hopping. By decreasing $\omega_{0}$ and therefore increasing the exciton-phonon coupling, it may be possible to induce self-localization of excitons. The dynamics of an excitation initially produced on a single molecule is illustrated in Figure 7. These results demonstrate that polar molecules trapped on an optical lattice can be used as a dynamically controllable simulator of open quantum systems.

\section{Conclusions}

The development of experimental techniques for cooling, trapping and controlling atoms and molecules has opened exciting opportunities for new studies of quantum many-body systems. Ultracold atoms and molecules are extensively researched as paradigm systems for engineering novel states of quantum matter and for quantum simulation of model Hamiltonians used in condensed matter physics. Most of these studies use ultracold atoms or 
molecules as probe particles. Here, we discuss the possibility of using collective excitations in an ensemble of molecules trapped on an optical lattice as a probe in a quantum simulation experiment.

We have shown that rotational excitations of polar molecules on an optical lattice may lead to the formation of collective many-body excitations - rotational Frenkel excitons. These excitons have unique properties that allow tuning the linear and non-linear exciton interactions by modifying the rotational structure of ultracold molecules by an external electric field. We suggest that this can be exploited for the study of new regimes of Frenkel exciton physics and the dynamics of quantum localization in disordered systems. We also suggest that rotational Frenkel excitons can be used for quantum simulation of the Holstein polaron model. This offers interesting possibilities to study quantum transport in open quantum systems with controllable interactions with the environment. In particular, the finite size and tunable properties of the phonon bath for molecules on an optical lattice suggests the possibility of exploring the transition from a non-Markovian to Markovian environment. To observe rotational excitons, one can measure the populations of the rotational states at different lattice sites. As described in Ref. [90], this can be achieved by applying a gradient of an electric field and detecting resonant transitions from Stark-shifted molecular levels.

\section{Acknowledgements}

We thank our collaborators, Felipe Herrera, Ping Xiang and Jesús Pérez-Ríos, who contributed to the original publications [59, 63, 64, 91] forming the basis of this article. Our

work is supported by NSERC of Canada and the Peter Wall Institute for Advanced Studies at the University of British Columbia.

\section{References}

[1] I. Bloch, J. Dalibard, and W. Zwerger, Rev. Mod. Phys. 80, 885 (2008)

[2] "Understanding Quantum Phase Transitions", editor: L. D. Carr (Taylor and Francis, Boca Raton, Fl, 2010)

[3] M. H. Anderson, J. R. Ensher, M. R. Matthews, C. E. Wieman, and E. A. Cornell, Science. 269, 198 (1995)

C. C. Bradley, C. A. Sackett, J. J. Tollett, and R. G. Hulet, Phys. Rev. Lett. 75, 1687 (1995)

K. B. Davis, M. O. Mewes, M. R. Andrews, N. J. van Druten, D. S. Durfee, D. M. Kurn, and W. Ketterle, Phys. Rev. Lett. 75, 3969 (1995)

E. A. Cornell and C. E. Wieman, Rev. Mod. Phys. 74, 875 (2002)

W. Ketterle, Rev. Mod. Phys. 74, 1131 (2002)

[4] M. R. Andrews, D. M. Kurn, H.-J. Miesner, D. S. Durfee, C. G. Townsend, S. Inouye, and W. Ketterle, Phys. Rev. Lett. 79, 553 (1997) 
[5] S. Trotzky, P. Cheinet, S. Fölling, M. Feld, U. Schnorrberger, A. M. Rey, A. Polkovnikov, E. A. Demler, M. D. Lukin and I. Bloch, Science. 319, 295 (2008)

[6] M. Anderlini, P. J. Lee, B. L. Brown, J. Sebby-Strabley, W. D. Phillips and J. V. Porto, Nature. 448, 452 (2007)

[7] T. Kraemer, M. Mark, P. Waldburger, J. G. Danzl, C. Chin, B. Engeser, A. D. Lange, K. Pilch, A. Jaakkola, H.-C. Nägerl and R. Grimm, Nature. 440, 315 (2006)

C. H. Greene, Physics Today. 63, 40 (2010)

[8] J. Kinast, S. L. Hemmer, M. E. Gehm, A. Turlapov, and J. E. Thomas, Phys. Rev. Lett. 92, 150402 (2004)

C. H. Schunck, Yong-il Shin, A. Schirotzek and W. Ketterle, Nature. 454, 739 (2008)

[9] C. A. Regal, M. Greiner, and D. S. Jin, Phys. Rev. Lett. 92, 040403 (2004)

M. Greiner, C. A. Regal, and D. S. Jin, Phys. Rev. Lett. 94, 070403 (2005)

C. A. Regal, M. Greiner, S. Giorgini, M. Holland, and D. S. Jin, Phys. Rev. Lett. 95, $250404(2005)$

[10] I. Bloch, Nature Physics. 1, 23 (2005)

[11] W. S. Bakr, J. I. Gillen, A. Peng, S. Fölling and M. Greiner, Nature. 462, 74 (2009)

W. S. Bakr, A. Peng, M. E. Tai, R. Ma, J. Simon, J. Gillen, S. Foelling, L. Pollet and M. Greiner, Science. 329, 547 (2010)

J. F. Sherson, C. Weitenberg, M. Endres, M. Cheneau, I. Bloch and S. Kuhr, Nature. 467, 68 (2010)

[12] M. Lewenstein, A. Sanpera, and V. Ahufinger, "Ultracold Atoms in Optical Lattices. Simulating quantum many-body systems" (Oxford University Press, USA, 2012)

M. Lewenstein, A. Sanpera, V. Ahufinger, B. Damski, A. Sen and U. Sen, Advances in Physics. 56, 243 (2007)

[13] M. Greiner, O. Mandel, T. Esslinger, T. W. Hänsch and I. Bloch, Nature. 415, 39 (2002)

[14] J. G. Bednorz and K. A. Müller, Z. Phys. B - Condensed Matter. 64, 189 (1986)

[15] L. D. Carr, D. DeMille, R. V. Krems and J. Ye, New Journal of Physics. 11, 055049 (2009)

[16] G. Pupillo, A. Micheli, H.P. Büchler, and P. Zoller, in "Cold Molecules: Theory, Experiment, Applications", editors: Roman Krems, Bretislav Friedrich and William C Stwalley (CRC Press, 2006)

[17] A. Micheli, G. K. Brennen and P. Zoller, Nature Physics. 2, 341 (2006)

R. Barnett, D. Petrov, M. Lukin, and E. Demler, Phys. Rev. Lett. 96, 190401 (2006)

G. K. Brennen, A. Micheli and P. Zoller, New J. Phys. 9, 138 (2007) 
H. P. Büuchler, A. Micheli, and P. Zoller, Nature Phys. 3, 726 (2007)

M. L. Wall and L. D. Carr, New J. Phys. 11, 055027 (2009)

C. Trefzger, M. Alloing, C. Menotti, F. Dubin and M. Lewenstein, New J. Phys. 12, 093008 (2010)

J. P. Kestner, B. Wang, J. D. Sau, and S. D. Sarma, Phys. Rev. B 83, 174409 (2011)

A. V. Gorshkov, S. R. Manmana, G. Chen, J. Ye, E. Demler, M. D. Lukin, and A. M. Rey, Phys. Rev. Lett. 107, 115301 (2011)

M. Lemeshko, R. V. Krems and H. Weimer, http://arxiv.org/abs/1203.0010

[18] M. Lewenstein, Nature physics. 2, 209 (2006)

[19] T. Volz, N. Syassen, D. M. Bauer, E. Hansis, S. Dürr and G. Rempe, Nature Physics. 2, $692(2006)$

A. Chotia, B. Neyenhuis, S. A. Moses, B. Yan, J. P. Covey, M. Foss-Feig, A. M. Rey, D. S. Jin and J. Ye, Phys. Rev. Lett. 108, 080405 (2012)

[20] S. Burger, K. Bongs, S. Dettmer, W. Ertmer, K. Sengstock, A. Sanpera, G. V. Shlyapnikov, and M. Lewenstein, Phys. Rev. Lett. 83, 5198 (1999)

U. Al Khawaja, H. T. C. Stoof, R. G. Hulet, K. E. Strecker, and G. B. Partridge, Phys. Rev. Lett. 89, 200404 (2002)

K. E. Strecker, G. B. Partridge, A. G. Truscott, and R. G. Hulet, Nature. 417, 150 (2002)

R. Balakrishnan, I. I. Satija and C. W. Clark, Phys. Rev. Lett. 103, 230403 (2009)

[21] D. H. J. O’Dell, S. Giovanazzi, and G. Kurizki, Phys. Rev. Lett. 90, 110402 (2003)

S. Sinha and G. V. Shlyapnikov, Phys. Rev. Lett. 94, 150401 (2005)

S. C. Cormack, D. Schumayer, and D. A. W. Hutchinson, Phys. Rev. Lett. 107, 140401 (2011)

[22] K. W. Madison, F. Chevy, W. Wohlleben, and J. Dalibard, Phys. Rev. Lett. 84, 806 (2000)

M. W. Zwierlein, J. R. Abo-Shaeer, A. Schirotzek, C. H. Schunck and W. Ketterle, Nature. 435, 1047 (2005)

A. Nunnenkamp, A. M. Rey and K. Burnett, Proc. R. Soc. A. 466, 1247 (2010)

[23] H. J. Lewandowski, D. M. Harber, D. L. Whitaker and E. A. Cornell, Phys. Rev. Lett. 88, $070403(2002)$

J. M. McGuirk J M, H. J. Lewandowski, D. M. Harber, T. Nikuni, J. E. Williams and E. A. Cornell, Phys. Rev. Lett. 89, 090402 (2002)

[24] A. Schirotzek, C.-H. Wu, A. Sommer, and M. W. Zwierlein, Phys. Rev. Lett. 102, $230402(2009)$

S. Nascimbène, N. Navon, K. J. Jiang, L. Tarruell, M. Teichmann, J. McKeever, F. Chevy, and C. Salomon, Phys. Rev. Lett. 103, 170402 (2009) 
C. Kohstall, M. Zaccanti, M. Jag, A. Trenkwalder, P. Massignan, G. M. Bruun, F. Schreck, and R. Grimm, http://arxiv.org/pdf/1112.0020.pdf

[25] R. E. Zillich and K. B. Whaley, Phys. Chem. Chem. Phys. 13, 18835 (2011)

[26] M. L. Wall and L. D. Carr, Phys. Rev. A. 82, 013611 (2010)

[27] K. A. Kuns, A. M. Rey and A. V. Gorshkov, Phys. Rev. A. 84, 063639 (2011)

[28] J. I. Cirac and P. Zoller, Nature Physics. 8, 264 (2012)

J. Buluta and F. Nori, Science. 326, 108 (2009)

[29] R. P. Feynman,International Journal of Theoretical Physics. 21, 467, 1982

[30] Yu. I. Manin, "Computable and non-computable" (Moscow, "Sovetskoe radio", 1980, p. 15), in Russian.

[31] I. Bloch, J. Dalibard and S. Nascimbène, Nature Physics. 8, 267 (2012)

[32] R. Blatt and C. F. Roos, Nature Physics. 8, 277 (2012)

[33] A. Aspuru-Guzik and P. Walther, Nature Physics. 8, 285 (2012)

[34] H. Weimer, M. Müller, I. Lesanovsky, P. Zoller and H. P. Büchler, Nature Physics. 6, $382(2010)$

[35] A. A. Houck, H. E. Türeci and J. Koch, Nature Physics. 8, 292 (2012)

[36] R. Hanson and D. D. Awschalom, Nature. 453, 1043 (2008)

[37] J. Simon, W. S. Bakr, R. Ma, M. E. Tai, P. M. Preiss and M. Greiner, Nature. 472, $307(2011)$

[38] M. Greiner and S. Fölling, Nature. 453, 736 (2008)

[39] C. Weitenberg, M. Endres, J. F. Sherson, M. Cheneau, P. Schauß, T. Fukuhara, I. Bloch, and S. Kuhr, Nature. 471, 319 (2011)

[40] F. H. L. Essler, H. Frahm, F. Göhmann, A. Klümper and V. E. Korepin, "The OneDimensional Hubbard Model" (Cambridge University Press, Cambridge, 2005) D. Jaksch, C. Bruder, J. I. Cirac, C. W. Gardiner and P. Zoller, Phys. Rev. Lett. 81, 3108 (1998)

M. Köhl, H. Moritz, T. Stöferle, K. Günter, T. Esslinger, Phys. Rev. Lett. 94, 080403 (2005)

[41] P. W. Anderson, P. A. Lee, M. Randeria, T. M. Rice, N. Trivedi and F. C. Zhang, J. Phys.: Condens. Matter. 16, R755 (2004)

[42] M. Lewenstein and A. Sanpera, Science. 319, 292 (2008)

[43] R. Micnas and B. Tobijaszewska, J. Phys.: Condens. Matter. 14, 9631 (2002) 
[44] H. Heiselberg, Phys. Rev. A. 73, 013628 (2006)

[45] W. S. Bakr, P. M. Preiss, M. Eric Tai, R. Ma, J. Simon and M. Greiner, Nature. 480, 5003 (2011)

[46] K. Góral, L. Santos and M. Lewenstein, Phys. Rev. Lett. 88, 170406 (2002)

[47] D. S. Jin and J. Ye, Physics Today. 64, 27 (2011)

[48] L. Tonks, Phys. Rev. 50, 955 (1936)

[49] M. Girardeau, J. Math. Phys. 1, 516, (1960)

[50] M. A. Cazalilla, R. Citro, T. Giamarchi, E. Orignac and M. Rigol, Rev. Mod. Phys. 83, 1405 (2011)

[51] R. Orbach, Phys. Rev. 112, 309 (1958)

[52] I. G. Kaplan, Theor. Math. Phys. 27, 466 (1976)

[53] V. M. Agranovich, "Excitations in Organic Solids" (Oxford University Press, Oxford, 2008)

[54] P. Jordan and E. Wigner, Z. Phys. 47, 631 (1928)

[55] T. Holstein and H. Primakoff, Phys. Rev. 58, 1098 (1940)

[56] D. B. Chesnut and A. Suna, J. Chem. Phys. 39, 146 (1963)

[57] V. M. Agranovich and B. S. Toshich, Zh. Eksp. Teor. Fiz. 53, 149 (1967)

[58] M. Litinskaya, Phys. Rev. B. 77, 155325 (2008)

[59] F. Herrera, M. Litinskaya and R. V. Krems, Phys. Rev. A. 82, 033428 (2010)

[60] J. Frenkel, Phys. Rev. 37, 17 (1931)

[61] V. M. Agranovich and D. M. Basko, J. Chem. Phys. 112, 8156 (2000)

[62] V. M. Agranovich and Yu. N. Gartstein, "Spatial dispersion and negative refraction of light", UFN (Physics: Uspekhi). 176, 1051 (2006)

[63] F. Herrera and R. V. Krems, Phys. Rev. A. 84, 051401(R) (2011)

[64] P. Xiang, M. Litinskaya and R. V. Krems, Phys. Rev. A 85, 061401(R) (2012)

[65] G. Vektaris, J. Chem. Phys. 101, 3031 (1994)

[66] P. Rabl and P. Zoller, Phys. Rev. A. 76, 042308 (2007)

[67] F. J. Dyson, Phys. Rev. 102, 1217 (1956)

[68] See for a review: A. Lagendijk, B. van Tiggelen and D. S. Wiersma, Physics Today. $62,24(2009)$ 
[69] D. S. Wiersma, P. Bartolini, A. Lagendijk and R. Righini, Nature. 390, 671 (1997)

[70] J. Billy, V. Josse, Z. Zuo, A. Bernard, B. Hambrecht, P. Lugan, D. Clément, L. Sanchez-Palencia, P. Bouyer and A. Aspect, Nature. 453, 891-894 (2008)

[71] J. Chabé, G. Lemarié, B. Grémaud, D. Delande, P. Szriftgiser and J. C. Garreau, Phys. Rev. Lett. 101, 255702 (2008)

[72] K. Binder and A. P. Young, Rev. Mod. Phys. 58, 801 (1986)

[73] N. Mason and A. Kapitulnik, Phys. Rev. Lett. 82, 5341 (1999)

N. Markovic, C. Christiansen, A. M. Mack, W. H. Huber and A. M. Goldman, arXiv:cond-mat/9904168v1.

[74] L. Levi, M. Rechtsman, B. Freedman, T. Schwartz, O. Manela and M. Segev, Science. $332,1541(2011)$

[75] J. M. Ziman, "Models of Disorder: The Theoretical Physics of Homogeneously Disordered Systems" (Cambridge University Press, 1979)

[76] I. M. Lifshitz, Zh. Eksp. Teor. Fiz. 18, 293 (1948)

I. M. Lifshitz and V. I. Peresada, Uchenye zapiski Kharkovskogo Universiteta Vol. 64; Trudy Fiz. otd. fiz.-mat. f-ta. 6, 37 (1955), in Russian

[77] O. A. Dubovsky and Yu. V. Konobeev, Fiz. Tverd. Tela (Leningrad). 7, 946 (1965)

[78] O. A. Dubovsky and Yu. V. Konobeev, Fiz. Tverd. Tela (Leningrad). 6, 946 (1965)

[79] H. Zoubi and G. C. La Rocca, Phys. Rev. B. 72, 125306 (2005)

[80] A. F. Ioffe and A. R. Regel, Prog. Semicond. 4, 237 (1960)

[81] G. Bergmann, Phys. Rep. 107, 1 (1984)

D. E. Khmelnitskii, Physica B (Amsterdam). 126, 235 (1984)

[82] H. Fukuyama and S. Hikami, "Anderson Localization” (Springer, Berlin, 1982)

[83] J. C. Flores, J. Phys. Cond. Matt. 1, 8471 (1989)

A. Bovier, J. Phys. A. 25, 1021 (1992)

[84] F. M. Izrailev and A. A. Krokhin, Phys. Rev. Lett. 82, 4062 (1999)

[85] L. Tessieri and F. M. Izrailev, Physica E. 9, 405 (2001)

[86] A. S. Alexandrov and N. F. Mott, "Polarons and Bipolarons" (World Scientific Publishing, Singapore, 1995)

[87] F. Caruso, A. W. Chin, A. Datta, S. F. Huelga and M. B. Plenio, J. Chem. Phys. 131, 105106 (2009)

P. Rebentrost, M. Mohseni, I. Kassal, S. Lloyd and A. Aspuru-Guzik, New J. Phys. 11, 033003 (2009)

D. Segal and D. R. Reichman, Phys. Rev. A. 76, 012109 (2007) 
[88] T. Holstein, Ann. Phys. (NY). 8, 325 (1959)

[89] W. P. Su, J. R. Schrieffer and A. J. Heeger, Phys. Rev. Lett. 42, 1698 (1979)

[90] D. DeMille, Phys. Rev. Lett. 88, 067901 (2002)

[91] J. Pérez-Ríos, F. Herrera and R. V. Krems, New J. Phys. 12, 103007 (2010) 